\title{
Planar QED at finite temperature and density: Hall conductivity, Berry's phases and minimal conductivity of graphene
}

\author{
C G Beneventano ${ }^{1}$, Paola Giacconi ${ }^{2}$, E M Santangelo ${ }^{1}$ \\ and Roberto Soldati ${ }^{2,3}$ \\ ${ }^{1}$ Departamento de Física and IFLP, Universidad Nacional de La Plata, CONICET, 1900 La Plata, \\ Argentina \\ ${ }^{2}$ Istituto Nazionale di Fisica Nucleare, Sezione di Bologna, I-40100 Bologna, Italy \\ ${ }^{3}$ Dipartimento di Fisica, Università di Bologna, Istituto Nazionale di Fisica Nucleare, \\ Sezione di Bologna, I-40126 Bologna, Italy
}

Received 4 January 2009, in final form 8 May 2009

Published 15 June 2009

Online at stacks.iop.org/JPhysA/42/275401

\begin{abstract}
We study one-loop effects for massless Dirac fields in two spatial dimensions, coupled to homogeneous electromagnetic backgrounds, both at zero and at finite temperature and density. In the case of a purely magnetic field, we analyze the relationship among the invariance of the theory under large gauge transformations, the appearance of Chern-Simons terms and of different Berry's phases. In the case of a purely electric background field, we show that the effective Lagrangian is independent of the chemical potential and of the temperature. More interesting, we show that the minimal conductivity, as predicted by the quantum field theory, is the right multiple of the conductivity quantum and is, thus, consistent with the value measured for graphene, with no extra factor of $\pi$ in the denominator.
\end{abstract}

PACS numbers: 11.10.Wx, 02.30.Sa, 73.43.-f

\section{Introduction}

Planar electrodynamics, i.e. electrodynamics in two spatial dimensions, has attracted attention for years, mainly due to its theoretical departure from what we are used to in three spatial dimensions - for two reviews of the subject, with emphasis on Chern-Simons terms, and many relevant references see $[1,2]$. A pedagogical introduction to planar electrodynamics can be found in [3].

In turn, more than 20 years ago, it was shown that, in the tight-binding approach, the effective theory at low momenta for a two-dimensional lattice of carbon atoms (which is now known as graphene) is nothing but a theory of massless Dirac fields in a $(2+1)$-dimensional 
Minkowski spacetime, in a reducible representation of the Clifford algebra (each irreducible representation corresponding to one Dirac point or valley) and with two fermion species, representing the spin of the interacting electrons $[4,5]$.

Stable monolayer samples of graphene were first isolated in 2004 [6]. The measurements, in 2005 , of the Hall conductivity in these samples confirmed that massless planar quantum electrodynamics is adequate for describing the effective behavior of quasi-particles in graphene [7]. The special, unique form for the conductivity of graphene, its many potential applications as well as the link that graphene establishes between a relativistic field theory and a condensed matter system, have triggered, during the last few years, a huge amount of work, both experimental and theoretical, on the subject—for a recent review, see [8].

In [9], we studied planar spinor electrodynamics at finite temperature and particle density, in the case of massless Dirac quantized fields in the presence of a homogeneous magnetic background. To this aim we evaluated, in the zeta function regularization approach [10], the one-loop effective action of the three-dimensional Euclidean theory, at finite temperature and in the presence of a real chemical potential. From such a result, we obtained the mean particle density, or equivalently the net charge, as a function of the temperature and of the chemical potential. The zero temperature limit of this charge, followed by a Lorentz boost, allowed us to obtain the Hall current as a function of the chemical potential. In particular, the behavior of the Hall conductivity around zero chemical potential was shown, in the same reference, to depend on the selection made for the so-called phase of the Dirac determinant [11] (for a discussion of this point, see also [12]). The present paper is a natural continuation of our previous work on the subject. Indeed, here we will study one-loop effective actions in the presence of those particular classical configurations characterizing the relevant experimental setups.

In sections 2 and 3, we present some basic conventions and calculations needed to study the massless theory in a purely magnetic homogeneous background, this time for a general complex-valued chemical potential. The introduction of an imaginary part in the chemical potential will allow us to discuss, in sections 4 and 5, the invariance of the effective theory under 'large' gauge transformations, their interpretation as $4 \pi$ rotations and the relationship among different phases of the determinant, the appearance (or lack thereof) of geometric phases at finite temperature as well as their interpretation as Chern-Simons terms or Berry's phases [13] in the zero temperature limit. These points were briefly discussed by two of us in the proceeding issue [14]. Here, we present a more detailed analysis of these topics.

Sections 6-8 treat the complementary case of a purely electric constant background field, both at zero and at non-vanishing, temperature and chemical potential. In section 6 , we use the Bogoliubov transformation method to study the vacuum persistence probability, determined by the imaginary part of the effective action at zero temperature, in the $(2+1)$-dimensional Minkowski spacetime. We obtain the probability of vacuum decay and show that, as expected for massless particles, it is far from being small. In section 7, we evaluate, through zeta function regularization, the effective action for the same background at finite temperature. We show that, in this case, the net charge is zero at all temperatures (equivalently, the effective action is independent of the chemical potential, whether real or complex). We also show that, in going back to Minkowski spacetime, the imaginary part of the effective action coincides, at all temperatures, with that obtained in the previous section in the zero temperature case.

The most impressive result in this paper is probably that contained in section 8 , where we evaluate, through the introduction of an adequate Lagrange multiplier, the mean current in the presence of a constant electric field. We show that such a calculation predicts a minimal conductivity which, at variance with many of the different results presented in the literature (see, for instance, $[15,16]$ ), is compatible with the measurements presented in [8]. Finally, 
section 9 contains our main conclusions, while the appendix presents a short overview of the main properties of the parabolic cylinder functions, needed to follow our calculation of the probability of vacuum decay at zero temperature.

\section{Dirac field interacting with a constant magnetic background at zero temperature}

In this section, we use the $2+1$ Minkowski spacetime metric $g^{\mu \nu}=\operatorname{diag}(+,-,-)$ and, throughout the paper (unless otherwise stated), we adopt natural units $\hbar=c=\tilde{c}=1$, where $\tilde{c}$ denotes the 'speed of light' for the two-dimensional system. Note that, for graphene, $\tilde{c}$ is approximately two orders of magnitude smaller than the speed of light in vacuum $[8,17]$. We choose the following representation for the Dirac matrices:

$$
\gamma^{0}=\sigma_{3}, \quad \gamma^{1}=\mathrm{i} \sigma_{1} \quad \text { and } \quad \gamma^{2}=\mathrm{i} \sigma_{2} .
$$

They satisfy the required properties

$$
\begin{aligned}
& \gamma^{\mu \dagger}=\gamma^{0} \gamma^{\mu} \gamma^{0}, \quad \gamma^{0}=\gamma^{0 \dagger}, \quad \gamma^{k}=-\gamma^{k \dagger}, \quad k=1,2 ; \\
& \left\{\gamma^{\mu}, \gamma^{\nu}\right\}=\gamma^{\mu} \gamma^{\nu}+\gamma^{\nu} \gamma^{\mu}=2 g^{\mu \nu} \mathbb{I} .
\end{aligned}
$$

As is well known [18], a second, nonequivalent representation of the Clifford algebra can be chosen, for instance, by reversing the sign of one of the spatial Dirac matrices. We will always consider massless fields, two fermion species or flavors, and the direct sum of both nonequivalent representations, i.e. the effective model for graphene $[4,5]$.

For the representation in equation (1), the one-particle Dirac Hamiltonian operator $H_{\mathrm{D}}$ is determined by the stationary solutions of the Dirac equation

$$
(\mathrm{i} \not \partial+e \not A) \Psi(x)=\not D \Psi(x)=0,
$$

where $-e$ is the negative electron charge. From the previous equation, it is easily seen that

$$
H_{\mathrm{D}}=-e A_{0}-\alpha^{k} D_{k},
$$

where $\gamma^{0} \gamma^{k}=\alpha^{k}=\left(-\sigma_{2}, \sigma_{1}\right)$ and $D_{k}=\mathrm{i} \partial_{k}+e A_{k}, k=1,2$.

If a uniform magnetic field is present along the positive $O z$-axis perpendicular to the Oxy-plane, after setting

$$
\Psi_{E}\left(x^{0}, \mathbf{x}\right)=\mathrm{e}^{-\mathrm{i} E x^{0}} \psi_{E}\left(x^{1}, x^{2}\right)=\mathrm{e}^{-\mathrm{i} E t} \psi_{E}(x, y),
$$

and choosing the Landau gauge $A^{\mu}=(0,0,-x B)$, with $B>0$, we get the one-particle Dirac Hamiltonian

$$
\begin{aligned}
H_{\mathrm{D}} & =\mathrm{i} \partial_{x} \sigma_{2}-\left(\mathrm{i} \partial_{y}+e B x\right) \sigma_{1} \\
& =\left(\begin{array}{cc}
0 & \partial_{x}-\mathrm{i} \partial_{y}-e B x \\
-\partial_{x}-\mathrm{i} \partial_{y}-e B x & 0
\end{array}\right) .
\end{aligned}
$$

We make the ansatz

$$
\psi_{E}(x, y)=\frac{1}{\sqrt{ } 2 \pi} \int_{-\infty}^{\infty} \mathrm{d} p \psi_{E, p}(x) \mathrm{e}^{\mathrm{i} p y},
$$

which allows us to determine the eigenvalues of the Hamiltonian from the set of equations

$$
\left(\begin{array}{cc}
E & -d_{x}-p+e B x \\
d_{x}-p+e B x & E
\end{array}\right) \psi_{E, p}(x)=0 .
$$

In order to find the energy eigenstates, it is convenient to introduce the dimensionless coordinate

$$
\xi=\sqrt{2 e B}\left(x-\frac{p}{e B}\right), \quad d_{x}=\sqrt{2 e B} d \xi .
$$


In terms of this variable, the one-particle Dirac Hamiltonian can be written as

$$
H_{\mathrm{D}}=\sqrt{2 e B}\left(\begin{array}{rr}
0 & \delta_{+} \\
-\delta_{-} & 0
\end{array}\right)
$$

with

$$
\delta_{ \pm}=d_{\xi} \mp \frac{\xi}{2}
$$

where the raising and lowering operators satisfy

$$
\begin{aligned}
& {\left[\delta_{ \pm}, \delta_{\mp}\right]= \pm 1, \quad\left[\delta_{\mp}, \delta_{\mp}\right]=0,} \\
& \delta_{+}|n\rangle=-|n+1\rangle, \quad \delta_{-}|n\rangle=n|n-1\rangle, \\
& \langle z \mid n\rangle=\phi_{n}(z), \quad\left\langle n^{\prime} \mid n\right\rangle=\delta_{n n^{\prime}} .
\end{aligned}
$$

Now, if we require the Dirac Hamiltonian to be self-adjoint on the domain $L^{2}\left(\mathbb{R}^{2}\right)$, we are driven to the orthonormal set of functions

$$
(n ! \sqrt{2 \pi})^{-1 / 2} D_{n}(\xi) \equiv \phi_{n}(\xi), \quad n=0,1,2, \ldots,
$$

where

$$
D_{n}(\xi)=2^{-n / 2} \mathrm{e}^{-\xi^{2} / 4} H_{n}(\xi / \sqrt{2})
$$

are parabolic cylinder functions of integer order. Note that the normalization constants have been chosen to satisfy

$$
\int_{-\infty}^{\infty} \mathrm{d} \xi \phi_{m}(\xi) \phi_{n}(\xi)=\delta_{m n}, \quad n, m=0,1,2, \ldots
$$

Hence, we obtain the following complete and orthonormal set of stationary improper eigenfunctions of the first-order Dirac Hamiltonian. These comprise an infinite number of modes with zero energy:

$$
\begin{aligned}
\Psi_{0, p}(t, x, y) & =\psi_{0, p}(\mathbf{x}) \quad \text { (Zero modes) } \\
& =\left(\frac{e B}{\pi}\right)^{1 / 4} \frac{1}{\sqrt{2 \pi}}\left(\begin{array}{l}
1 \\
0
\end{array}\right) \exp \left\{\mathrm{i} p y-\frac{1}{4} \xi^{2}\right\},
\end{aligned}
$$

and an infinite number of modes with nonvanishing energies,

$$
\begin{aligned}
\Psi_{n, p}^{( \pm)}(t, x, y) & =\psi_{n, p}^{( \pm)}(\mathbf{x}) \exp \left\{ \pm \mathrm{i} E_{n} t\right\} \\
& =\left(\frac{e B}{2}\right)^{1 / 4} \frac{1}{\sqrt{2 \pi}} \exp \left\{ \pm \mathrm{i} E_{n} t+\mathrm{i} p y\right\}\left(\begin{array}{c}
\phi_{n}(\xi) \cos \theta_{n}^{ \pm} \\
\phi_{n-1}(\xi) \sin \theta_{n}^{ \pm}
\end{array}\right),
\end{aligned}
$$

corresponding to eigenenergies $\mp E_{n}=\mp \sqrt{2 n e B}$, where $\tan \theta_{n}^{ \pm}=\mp E_{n}$.

The spectrum is purely discrete and each eigenvalue exhibits the well-known continuous Landau degeneracy per unit area,

$$
\Delta=\frac{e B}{2 \pi} .
$$

In fact, the improper Dirac eigenspinors are normalized according to

$$
\begin{aligned}
& \int \mathrm{d} \mathbf{x} \psi_{0, l}^{\dagger}(\mathbf{x}) \psi_{0, k}(\mathbf{x})=\delta(k-l) \\
& \int \mathrm{d} \mathbf{x} \bar{\psi}_{m, l}^{( \pm)}(\mathbf{x}) \gamma^{0} \psi_{n, k}^{( \pm)}(\mathbf{x})=\delta_{m n} \delta(l-k)
\end{aligned}
$$


and, as a consequence, we have

$$
\begin{aligned}
& \int_{-\infty}^{\infty} \mathrm{d} k \psi_{0, k}^{\dagger}(\mathbf{x}) \psi_{0, k}(\mathbf{x})=\Delta, \\
& \int_{-\infty}^{\infty} \mathrm{d} k \bar{\psi}_{n, k}^{( \pm)}(\mathbf{x}) \gamma^{0} \psi_{n, k}^{( \pm)}(\mathbf{x})=\Delta, \quad \forall n \in \mathbb{N} .
\end{aligned}
$$

The corresponding eigenfunctions in the other nonequivalent representation, where $\gamma^{1} \rightarrow-\gamma^{1}$, are obtained through $\psi_{0, k} \rightarrow \sigma_{1} \psi_{0, k}, \psi_{n, k} \rightarrow \sigma_{1} \psi_{n, k}$, and the corresponding energies are the same as those found in the present representation. Finally, to describe graphene, an overall degeneracy of 2 (two species or 'flavors') must be considered to take the spin of the electrons in the original tight binding model into account.

\section{Dirac field interacting with a constant magnetic background, at finite temperature and density}

In order to consider the effect of temperature, we study the same problem as in the previous section, this time in the Euclidean three-dimensional space, by considering the effective oneloop action in the path integral approach. This requires the evaluation of the determinant of the corresponding Euclidean Dirac operator, through some regularization method, which we will choose to be the zeta function one [10]. To this end, we take the Euclidean gamma matrices in one of the two nonequivalent representations as

$$
\bar{\gamma}_{j}=-\mathrm{i} \gamma^{j}, \quad j=1,2, \quad \bar{\gamma}_{3}=\gamma^{0},
$$

so that

$$
\bar{\gamma}_{\mu}=\sigma_{\mu}, \quad \mu=1,2,3, \quad\left\{\bar{\gamma}_{\mu}, \bar{\gamma}_{\nu}\right\}=2 \delta_{\mu \nu} \mathbb{I}
$$

and set

$$
\mathrm{i} x_{0}=x_{3}, \quad x_{\mu}=\left(x_{1}, x_{2}, x_{3}\right)=(\mathbf{x}, \tau)=(x, y, \tau),
$$

together with

$$
A_{0}=\mathrm{i} A_{3}, \quad \bar{A}_{\mu}=\left(\mathbf{A}, A_{3}\right)=(0, B x,-\mathrm{i} v / e),
$$

where a generalized complex chemical potential $v=\mu+\mathrm{i} \gamma$ has been introduced. Note that the presence of a real chemical potential in the Minkowski spacetime just amounts to a trivial overall shift of the energy scale. At finite temperature, instead, the derivative of the effective action with respect to the real chemical potential will give us the mean value of the net charge of the theory. The imaginary part of the chemical potential will, later on, allow us to interpret our results in terms of topological phases.

To describe graphene, we treat the massless Euclidean Dirac operator

$$
\begin{aligned}
(\not D)_{\text {Eucl }} & =\sigma_{1}\left(-\mathrm{i} \partial_{x}\right)+\sigma_{2}\left(-\mathrm{i} \partial_{y}-e B x\right)+\sigma_{3}\left(-\mathrm{i} \partial_{\tau}+\mathrm{i} v\right) \\
& =\sigma_{\lambda}\left(p_{\lambda}-e B x \delta_{2 \lambda}-\gamma \delta_{3 \lambda}\right)+\mathrm{i} \mu \sigma_{3} .
\end{aligned}
$$

In order to evaluate the functional determinant, we look for the spectral resolution of (22), which is not a self-adjoint operator for $\mu=\operatorname{Re} v \neq 0$. We restrict its domain to regular square summable functions in $x$. Moreover, we shall adopt antiperiodic boundary conditions on the strip $0 \leqslant \tau \leqslant \beta$, where $\beta=1 / T$ (note that $k_{B}$ has been put to 1 in natural units). As a consequence of this antiperiodicity, the three-dimensional Euclidean space manifold is the direct product $\mathcal{M}_{3}=\mathbb{R}^{2} \times C^{1}\left(\frac{\beta}{2 \pi}\right)$, where $C^{n}(r)$ is the circumference of radius $r$ and integer winding number equal to $n \in \mathbb{Z}$. Hence, the symmetry group of $\mathcal{M}_{3}$ becomes the direct 
product $U(1) \times O(2) \doteq U(1) \times U(1)$, that is, the square of the unitary, non-simply connected, Abelian group $U(1)$.

To satisfy the antiperiodicity, we introduce the Matsubara angular frequencies

$$
\omega_{\ell}=(2 \ell+1) \frac{\pi}{\beta}, \quad \ell \in \mathbb{Z}
$$

and propose, for the eigenfunctions of (22),

$$
\psi(x, y, \tau)=\frac{1}{\sqrt{ } 2 \pi \beta} \sum_{\ell, p} \exp \left\{\mathrm{i}\left(\tau \omega_{\ell}+p y\right)\right\} \psi_{\ell, p}(x),
$$

where

$$
y_{\ell, p} \equiv \sum_{\ell=-\infty}^{\infty} \int_{-\infty}^{\infty} \mathrm{d} p
$$

Thus, the problem to be solved becomes

$$
\left(\begin{array}{cc}
\omega_{\ell}+\mathrm{i} v-\lambda & -\mathrm{i} d_{x}+\mathrm{i}(e B x-p) \\
-\mathrm{i} d_{x}-\mathrm{i}(e B x-p) & -\omega_{\ell}-\mathrm{i} v-\lambda
\end{array}\right)\left(\begin{array}{l}
\varphi_{\ell, p} \\
\chi_{\ell, p}
\end{array}\right)=0 .
$$

The resulting spectrum is complex and consists of two parts. We will call the first part asymmetric, since, given one eigenvalue $\lambda$ in this part of the spectrum, $-\lambda$ does not belong to the spectrum. The second part, which we will call the symmetric part of the spectrum, instead, is such that, for each eigenvalue $\lambda$ belonging to this part of the spectrum, $-\lambda$ also belongs to the spectrum. (Note that this last part behaves as expected from the eigenvalues of a square root operator.)

(i) The asymmetric piece of the spectrum, which is an infinite 'tower' of states associated with the lowest Landau level in the Hamiltonian, is given by

$$
\lambda_{\ell}=\omega_{\ell}+\mathrm{i} v=\omega_{\ell}-\gamma+\mathrm{i} \mu \quad(\ell \in \mathbb{Z})
$$

and has a corresponding set of eigenfunctions

$$
\Psi_{\ell, p}(x, y, \tau)=\left(\frac{e B}{\pi}\right)^{\frac{1}{4}}\left(\begin{array}{c}
\mathrm{e}^{-\frac{1}{2} e B(x-p / e B)^{2}} \\
0
\end{array}\right) \exp \left\{\mathrm{i} \tau \omega_{\ell}+\mathrm{i} p y\right\}(2 \pi \beta)^{-\frac{1}{2}} .
$$

Their normalization is given by

$$
\int_{0}^{\beta} \mathrm{d} \tau \int_{-\infty}^{\infty} \mathrm{d} x \int_{-\infty}^{\infty} \mathrm{d} y \Psi_{\ell, p}(x, y, \tau) \Psi_{\jmath, q}^{*}(x, y, \tau)=\delta_{\ell_{\jmath}} \delta(p-q)
$$

and the degeneracy per unit area is, as in the zero temperature case,

$$
\int_{0}^{\beta} \mathrm{d} \tau \int_{-\infty}^{\infty} \mathrm{d} p\left|\Psi_{\ell, p}(x, y, \tau)\right|^{2}=\frac{e B}{2 \pi}=\Delta .
$$

(ii) The symmetric part of the spectrum is related to the excited Landau levels. It is given by

$$
\lambda= \pm \lambda_{\ell, n}, \quad \lambda_{\ell, n}=\sqrt{\lambda_{\ell}^{2}+2 e B n,}
$$

with $n \in \mathbb{N}$ and $\ell \in \mathbb{Z}$. To obtain the corresponding eigenstates, it is convenient to set

$$
z=x \sqrt{2 e B}-p \sqrt{\frac{2}{e B}}, \quad d_{x}=\sqrt{2 e B} d_{z}
$$

so that

$$
d_{x} \pm(p-e B x)=\sqrt{2 e B}\left(d_{z} \mp \frac{z}{2}\right) \equiv \sqrt{2 e B} \delta_{ \pm},
$$


where the raising-lowering operators satisfy

$$
\begin{aligned}
& {\left[\delta_{ \pm}, \delta_{\mp}\right]= \pm 1, \quad\left[\delta_{\mp}, \delta_{\mp}\right]=0,} \\
& \delta_{+}|n\rangle=-|n+1\rangle, \quad \delta_{-}|n\rangle=n|n-1\rangle, \\
& \langle z \mid n\rangle=\phi_{n}(z), \quad\langle m \mid n\rangle=\delta_{m n}, \\
& \phi_{n}(z) \equiv(n ! \sqrt{2 \pi})^{-\frac{1}{2}} D_{n}(z), \quad n=1,2, \ldots
\end{aligned}
$$

Hence, if we rewrite the Euclidean Dirac operator in the form

$$
(\not D)_{\mathrm{Eucl}}=\sqrt{2 e B}\left(\begin{array}{cc}
\lambda_{\ell} & -\mathrm{i} \delta_{+} \\
-\mathrm{i} \delta_{-} & -\lambda_{\ell}
\end{array}\right)
$$

where $\lambda_{\ell}=\lambda_{\ell} / \sqrt{2 e B}$, it turns out that the normalized eigenstates are given by

$\Psi_{\ell, p, n, \pm}=(2 e B)^{\frac{1}{4}} \exp \left\{\mathrm{i} \tau \omega_{\ell}+\mathrm{i} p y\right\}(4 \pi \beta)^{-\frac{1}{2}}\left(1+\left|\varkappa_{\ell, n, \pm}\right|^{2}\right)^{-\frac{1}{2}}\left(\begin{array}{c} \pm \mathrm{i} \phi_{n}(z) \\ \varkappa_{\ell, n, \pm} \phi_{n-1}(z)\end{array}\right)$,

with

$$
\varkappa_{\ell, n, \pm}=\frac{1}{\sqrt{ } 2 e B}\left(\lambda_{\ell, n} \mp \lambda_{\ell}\right)=\frac{n \sqrt{2 e B}}{\lambda_{\ell, n} \pm \lambda_{\ell}} .
$$

Moreover, by setting

$$
\lambda_{\ell, n} \equiv \rho_{\ell, n}\left(\cos \alpha_{\ell, n}+\mathrm{i} \sin \alpha_{\ell, n}\right),
$$

one finds

$$
\begin{aligned}
& \rho_{\ell, n}=\frac{1}{\sqrt{ } 2 e B}\left\{\left[\left(\omega_{\ell}-\gamma\right)^{2}-\mu^{2}+2 e B n\right]^{2}+4 \mu^{2}\left(\omega_{\ell}-\gamma\right)^{2}\right\}^{\frac{1}{4}}, \\
& \tan 2 \alpha_{\ell, n}=\frac{2 \mu\left(\omega_{\ell}-\gamma\right)}{\left(\omega_{\ell}-\gamma\right)^{2}-\mu^{2}+2 e B n}
\end{aligned}
$$

and thereby

$$
\begin{aligned}
& \operatorname{Re} \varkappa_{\ell, n, \pm}=\rho_{\ell, n} \cos \alpha_{\ell, n} \mp\left(\omega_{\ell}-\gamma\right), \\
& \operatorname{Im} \varkappa_{\ell, n, \pm}=\rho_{\ell, n} \sin \alpha_{\ell, n} \mp \mu .
\end{aligned}
$$

It might be convenient to introduce another different parametrization for the eigenspinors, which makes use of a pair of sequences of angles, namely

$$
0<\theta_{\ell, n}^{ \pm}<\frac{\pi}{2}, \quad 0<\Theta_{\ell, n}^{ \pm}<2 \pi \quad(\ell \in \mathbb{Z}, n \in \mathbb{N}) .
$$

Thus, after calling

$$
\varkappa_{\ell, n, \pm} \equiv \tan \theta_{\ell, n}^{ \pm} \exp \left\{\mathrm{i} \Theta_{\ell, n}^{ \pm}\right\},
$$

we can also write

$$
\Psi_{\ell, p, n}^{ \pm}=\frac{(2 e B)^{\frac{1}{4}}}{\sqrt{ } 4 \pi \beta} \exp \left\{\mathrm{i} \tau \omega_{\ell}+\mathrm{i} p y\right\}\left(\begin{array}{c} 
\pm \mathrm{i} \phi_{n}(z) \cos \theta_{\ell, n}^{ \pm} \\
\exp \left\{\mathrm{i} \Theta_{\ell, n}^{ \pm}\right\} \phi_{n-1}(z) \sin \theta_{\ell, n}^{ \pm}
\end{array}\right) .
$$

Also in this case, the degeneracy per unit area is given by the Landau factor $\Delta$ in (18). We remark that, when going to the other nonequivalent representation, the symmetric part of the spectrum remains the same, but the asymmetric part transforms according to $\lambda_{\ell} \rightarrow-\lambda_{\ell}$ or, equivalently, $v \rightarrow-v$. 


\section{The effective action. Invariance under large gauge transformations in the case of a complex chemical potential}

It is important to note that, as can be seen from (24) and (26), the whole spectrum is, in any of the two representations of the Clifford algebra, invariant under the so-called large gauge transformations $[1,19]$ :

$$
v \rightarrow v^{\prime}=v+\frac{2 \pi \mathrm{i} J}{\beta}, \quad \psi(\mathbf{x}) \rightarrow \psi^{\prime}(\mathbf{x})=\psi(\mathbf{x}) \mathrm{e}^{\mathrm{i} \tau \frac{2 \pi J}{\beta}} \quad(J \in \mathbb{Z}),
$$

where $\mathbf{x}=(x, y, \tau)$, which are precisely those Euclidean gauge transformations leaving untouched the antiperiodic boundary conditions satisfied by the spinor Euclidean wavefunctions and, in consequence, the Fermi-Dirac statistics. In other words, the discrete imaginary shift of the chemical potential $\beta v \rightarrow \beta v+2 \pi \mathrm{i} J(J \in \mathbb{Z})$ is a symmetry transformation of the theory. In contrast, any other imaginary shift of the chemical potential cannot be compensated by a phase transformation of the Euclidean spinor wavefunction, for the latter one would spoil the antiperiodic boundary conditions that the Euclidean spinor field must satisfy in order to have the right statistics.

The Euclidean effective action for a sample of area $\Omega$, as defined in terms of the zeta function regularization technique [10], is given by

$$
\begin{aligned}
\Gamma_{\text {eff }} & =\ln Z \equiv \ln \operatorname{det}\left(\ell_{0}(\not D)_{\text {Eucl }}\right) \\
& \equiv\left[-\frac{\mathrm{d}}{\mathrm{d} s} \zeta\left(s, \not D \ell_{0}\right)\right]_{s=0} \\
& =-\zeta^{\prime}\left(0, \not D \ell_{0}\right) \\
& =\Omega \Delta[A(a)+S(a, b)] .
\end{aligned}
$$

In the last equation, we used the dimensionless reduced variables

$$
a \equiv \frac{\beta v}{2 \pi}, \quad b \equiv \frac{1}{2} \beta \sqrt{2 e B},
$$

the arbitrary length scale $\ell_{0}$ being introduced to render the argument of the zeta function dimensionless. It is important to stress that physically meaningful results must be independent of $\ell_{0}$. Here, $A$ and $S$ denote the dimensionless reduced contributions to the full effective action that originate respectively from the asymmetric and from the symmetric parts of the spectrum of the Euclidean Dirac operator respectively.

In [9], we presented a detailed calculation of the effective action in the case of a purely real chemical potential $(v=\mu)$, and showed that different selections of the cut in the complex plane of the eigenvalues of (22) during the evaluation of the determinant lead to predictions for the Hall conductivity which reproduce the behavior measured for mono- and bilayer samples. In this section, we will concentrate on the contribution to the effective action due to the asymmetric portion of the spectrum in the case of a complex chemical potential-a survey of this calculation appears in [14].

The contribution to the effective action coming from the symmetric part of the spectrum does not suffer from regularization ambiguities. It is given by

$$
S(a, b)=2 b \zeta_{\mathrm{R}}\left(-\frac{1}{2}\right)+\sum_{n=1}^{\infty} \ln \left\{\left(1+z \mathrm{e}^{-\beta \varepsilon_{n}}\right)\left(1+z^{-1} \mathrm{e}^{-\beta \varepsilon_{n}}\right)\right\},
$$

where $z=\mathrm{e}^{2 \pi a}, \varepsilon_{n} \equiv \sqrt{2 e B n}$ and $\zeta_{\mathrm{R}}$ is Riemann's zeta function. Note that this expression is invariant under the discrete symmetry $v \rightarrow-v$, which is a symmetry of this portion of the spectrum. 
On the other hand, the contribution to the effective action arising from the asymmetric part of the spectrum is given by

$$
A(a)=-\zeta_{A}^{\prime}(0, a),
$$

with

$$
\begin{aligned}
\zeta_{A}(s, a) & =\left(\frac{2 \pi \ell_{0}}{\beta}\right)^{-s} \sum_{\ell=0}^{\infty}\left[\left(\ell+\frac{1}{2}+\mathrm{i} \frac{\beta v}{2 \pi}\right)^{-s}+\left(-\ell-\frac{1}{2}+\mathrm{i} \frac{\beta v}{2 \pi}\right)^{-s}\right] \\
& =\left(\frac{2 \pi \ell_{0}}{\beta}\right)^{-s} \sum_{\ell=0}^{\infty}\left[\left(\ell+q_{+}\right)^{-s}+\mathrm{e}^{\mp \mathrm{i} \pi s}\left(\ell+q_{-}\right)^{-s}\right] .
\end{aligned}
$$

The right-hand side involves the sum of two series representations of Hurwitz zeta functions, $\zeta(s, q)$, which are well defined iff

$$
\operatorname{Re} s>1, \quad \beta \gamma \neq \pm \pi(2 n-1) \quad(n \in \mathbb{N}) .
$$

In (36), we have introduced the complex quantities

$$
q_{ \pm} \equiv \frac{1}{2} \pm \frac{\mathrm{i} \beta}{2 \pi}(\mu+\mathrm{i} \gamma), \quad-\pi<\beta \gamma<\pi .
$$

The regularization ambiguity, which gives rise to a phase of the determinant [11], has its roots in the factor $\mathrm{e}^{\mp \mathrm{i} \pi s}$ or, equivalently, in the selection of the cut of the complex power in the plane of the eigenvalues of (22). Selecting one or the other sign (cut in the lower (upper) complex plane), irrespective of the sign of $\mu$, we get

$$
\zeta_{A}(s, a)=\left(\frac{2 \pi \ell_{0}}{\beta}\right)^{-s}\left\{\zeta\left(s, q_{+}\right)+\mathrm{e}^{\mp \mathrm{i} \pi s} \zeta\left(s, q_{-}\right)\right\} .
$$

For the contribution to the effective action we obtain, after evaluating the derivative,

$$
\begin{aligned}
A(a) & =\ln 2 \pi-\ln \Gamma\left(q_{+}\right) \Gamma\left(q_{-}\right) \pm \mathrm{i} \pi\left(\frac{1}{2}-q_{-}\right) \\
& =\ln 2 \pi-\ln \Gamma\left(\frac{1}{2}+\mathrm{i} a\right) \Gamma\left(\frac{1}{2}-\mathrm{i} a\right) \mp \pi a, \quad \text { for } \quad-\pi<\beta \gamma<\pi, \quad \mu \in \mathbb{R} .
\end{aligned}
$$

The previous result can also be cast in the form

$$
\begin{aligned}
A(a) & =\ln 2 \cosh \pi a \mp \pi a \\
& =\ln \left(\mathrm{e}^{\pi a}+\mathrm{e}^{-\pi a}\right) \mp \pi a \\
& =\ln \left[\mathrm{e}^{\frac{1}{2} \beta(\mu+\mathrm{i} \gamma)}+\mathrm{e}^{-\frac{1}{2} \beta(\mu+\mathrm{i} \gamma)}\right] \mp \frac{1}{2} \beta(\mu+\mathrm{i} \gamma) .
\end{aligned}
$$

So, in our case, a well-known sign ambiguity [11, 19] appears when evaluating the determinant of the Dirac operator, as it happens whenever this last operator is not the square root of a second-order operator of the Laplace type. Note that if the so-called phase of the determinant were chosen with the same sign for all values of $\mu$, then the contribution of each representation to the effective action would not be an even function of $\mu$ for $\gamma=0$. As a consequence, the contribution to the charge due to each Dirac point would not change sign as $\mu \rightarrow-\mu$.

Now, it is always possible and convenient (although not mandatory) to relate the two opposite phase prescriptions to the sign of the (real part of) the chemical potential.

Turning back to equation (36), if we cut the complex plane of the eigenvalues $\lambda_{\ell}^{ \pm}=\left(\ell+q_{ \pm}\right)(\ell=0,1,2, \ldots)$ along the upper half-plane for positive values of $\mu$ and along the lower half-plane for negative values of $\mu$, for example, we obtain what we will call the standard prescription, and will identify through the index $\kappa=-1$. In this case, we have

$$
A(a)-\ln 2 \cosh \pi a=\left\{\begin{array}{ll}
-\pi a, & \text { for } \mu>0 \\
+\pi a, & \text { for } \mu<0
\end{array} \quad(\kappa=-1) .\right.
$$


We shall call the non-standard prescription, $\kappa=+1$, the opposite one:

$$
A(a)-\ln 2 \cosh \pi a=\left\{\begin{array}{ll}
+\pi a, & \text { for } \mu>0 \\
-\pi a, & \text { for } \mu<0
\end{array} \quad(\kappa=+1) .\right.
$$

In so doing, for $\gamma=0$, we recover our previous result [9], namely

$$
\frac{1}{\Omega} \Gamma_{\text {eff }}=\Delta\left\{\frac{1}{2} \kappa \beta|\mu|+\ln 2 \cosh \frac{\beta \mu}{2}+2 b \zeta\left(-\frac{1}{2}\right)\right\}+\Delta \sum_{n=1}^{\infty} \ln \left\{\left(1+z \mathrm{e}^{-\beta \varepsilon_{n}}\right)\left(1+z^{-1} \mathrm{e}^{-\beta \varepsilon_{n}}\right)\right\} .
$$

These two criteria lead, for a complex chemical potential such that $-\pi<\beta \gamma<\pi$, to a contribution of the asymmetric part of the spectrum to the effective action, which is given by

$$
\begin{aligned}
A(a) & =\ln \left(\mathrm{e}^{\pi a}+\mathrm{e}^{-\pi a}\right)+\pi a \kappa \operatorname{sgn}(\mu) \\
& =\ln \left[\mathrm{e}^{\frac{1}{2} \beta(\mu+\mathrm{i} \gamma)}+\mathrm{e}^{-\frac{1}{2} \beta(\mu+\mathrm{i} \gamma)}\right]+\frac{1}{2} \kappa \beta(\mu+\mathrm{i} \gamma) \operatorname{sgn}(\mu) .
\end{aligned}
$$

This contribution turns out to be invariant under the generalized conjugation symmetry $a \rightarrow-a$.

One can take the limits $\beta \gamma \rightarrow \pm \pi$ in the last expression and write

$$
\begin{aligned}
& \lim _{\beta \gamma \rightarrow \pi^{-}} A(a)=\ln 2 \sinh \frac{\beta|\mu|}{2}+\frac{1}{2} \kappa \beta|\mu|+\frac{\pi \mathrm{i}}{2}(1+\kappa) \operatorname{sgn}(\mu), \\
& \lim _{\beta \gamma \rightarrow-\pi^{+}} A(a)=\ln 2 \sinh \frac{\beta|\mu|}{2}+\frac{1}{2} \kappa \beta|\mu|-\frac{\pi \mathrm{i}}{2}(1+\kappa) \operatorname{sgn}(\mu),
\end{aligned}
$$

and therefore

$$
\left[\lim _{\beta \gamma \rightarrow \pi^{-}}-\lim _{\beta \gamma \rightarrow-\pi^{+}}\right] A(a)=\left\{\begin{array}{cl}
0 & \text { for } \quad \kappa=-1 \\
2 \pi \mathrm{i} \operatorname{sgn}(\mu) & \text { for } \quad \kappa=+1
\end{array}\right.
$$

However, the exact calculation can be performed at both extreme points of the interval, and both results coincide, as dictated by the invariance of the spectrum under large gauge transformations, which amounts to $\beta \gamma \rightarrow \beta \gamma+2 \pi$. The zeta function regularization, which is based solely on the properties of the spectrum, respects this invariance for all values of $\beta \gamma$. In a zeta function regularization approach, it is not only the partition function that is invariant under these transformations but it is the effective action itself that remains invariant. So, we can limit ourselves to give the result for the contribution to the effective action of the asymmetric part of the spectrum, for $-\pi<\beta \gamma \leqslant \pi$,

$$
\begin{aligned}
\Omega^{-1} \Gamma_{\mathrm{eff}}= & \Delta[A(a)+S(a, b)] \\
= & \frac{e B}{2 \pi}\left\{\ln \left(\mathrm{e}^{\pi a}+\mathrm{e}^{-\pi a}\right)+\pi a \kappa \operatorname{sgn}(\mu)+2 b \zeta\left(-\frac{1}{2}\right)\right. \\
& \left.+\sum_{n=1}^{\infty} \ln \left[\left(1+z \mathrm{e}^{-\beta \varepsilon_{n}}\right)\left(1+z^{-1} \mathrm{e}^{-\beta \varepsilon_{n}}\right)\right]\right\} .
\end{aligned}
$$

The result for any other value can be obtained by using

$$
\Gamma_{\text {eff }}(\beta \mu+\mathrm{i} \beta \gamma)=\Gamma_{\text {eff }}(\beta \mu+2 \pi \mathrm{i} j+\mathrm{i} \beta \gamma) \quad(j \in \mathbb{Z}) .
$$

In fact, for any of the two selections of $\kappa$, the whole effective action, per unit degree of freedom, is invariant under large gauge transformations. As explained in [14], this amounts to an invariance under rotations of angle $4 \pi$ around an axis perpendicular to the plane of the sample in the Euclidean space. We discuss this point in the following section. 


\section{Zero temperature limit}

Owing to the fact that the eigenfunctions corresponding to the asymmetric part of the spectrum are eigenstates of $\sigma_{3}$ with eigenvalue +1 , one can equivalently write gauge transformations in the form

$$
\Psi_{\ell, p}(x, y, \tau) \rightarrow \exp \left\{\frac{1}{2} \mathrm{i} \sigma_{3}(2 \gamma) \tau\right\} \Psi_{\ell, p}(x, y, \tau) .
$$

This last expression shows that, as the Euclidean time $\tau$ grows from 0 to $\beta$, spinors are rotated by $2 \beta \gamma$, since $\frac{1}{2} \sigma_{3}$ is the generator of rotations in the $O x y$-plane. In particular, $\beta \gamma=2 \pi$ corresponds to a rotation of angle $4 \pi$ around the magnetic field. Such a rotation must be an invariance for spinors, which provides one interpretation of the invariance under large gauge transformations.

On the other hand, $\beta \gamma=\pi$ corresponds to a rotation of angle $2 \pi$. As can be seen from (43), at finite temperature, such a transformation turns the statistics from Fermi-Dirac to BoseEinstein, for any of the two selections of phases in one of the two inequivalent representations (for a discussion about Bose-Fermi transmutation and relativistic 'anyons' see, for instance, [20]). For $\kappa=+1$, it also gives rise to an overall phase of $\pi$ per unit degeneracy in the partition function, which can be recognized as Berry's phase. Such a phase is the contribution which survives in the low temperature limit. In fact, in this limit one has from equation (42)

$$
\Omega \Delta A(a)=\frac{1}{2}(\kappa+1) \Omega \Delta \beta(\mu+\mathrm{i} \gamma) \operatorname{sgn}(\mu) \quad(\beta \rightarrow \infty)
$$

Thus, in the zero temperature limit, the effective action vanishes for the standard phase prescription $(\kappa=-1)$, so that the partition function is rotationally invariant. For $\kappa=+1$, the imaginary term in $\Omega \Delta A(a)$ (i.e. the one proportional to $\gamma$ ) is the Abelian Chern-Simons term:

$$
\mathrm{i} \beta \gamma \Omega \frac{e B}{2 \pi} \operatorname{sgn}(\mu)=\mathcal{A}_{\mathrm{CS}}=\frac{\beta \Omega}{2} \kappa_{\mathrm{CS}} \frac{\mathrm{i} e^{2}}{2 \pi} \varepsilon^{\mu \nu \rho} F_{\mu \nu} A_{\rho},
$$

where $\kappa_{\mathrm{CS}}$ denotes the Chern-Simons coefficient, a pure number of a topological origin (remember that $\mathrm{i} e A_{3}=\mu+\mathrm{i} \gamma$ ). Our calculation clearly shows that the latter one is invariant, as expected, under large gauge transformations, which represent rotations by integer multiples of $4 \pi$. In contrast, under rotations of angle $2 \pi(\beta \gamma=\pi)$ the partition function is multiplied by (when taking into account the two flavors)

$$
Z(\beta \gamma=\pi) \rightarrow Z \mathrm{e}^{ \pm 2 \pi \mathrm{i} \Omega \Delta} \text {. }
$$

Hence, the requirement of the partition function, in each representation, being invariant under rotations of angle $2 \pi$, for an area $\Omega$, entails a quantization condition on the coefficient of the Chern-Simons term or, equivalently, on the reduced flux of the magnetic field, i.e. $\Omega \Delta=N \in \mathbb{N}$. Interestingly enough, this is precisely the condition for physical states to transform according to the one-dimensional ray representations of the magnetic translation group [21].

Also note that the geometric phase appearing for $\kappa=+1$ leads, in the zero temperature limit, to Berry's phase which equals $\pi$ for each degree of freedom. A similar calculation in the other irreducible representation of the Clifford algebra would give exactly the same results, if the same criterion were used to fix the phase of the determinant. As stressed in [9], the right behavior for the Hall conductivity in monolayer graphene appears when choosing opposite criteria in the two nonequivalent representations, which corresponds to total Berry's phase of $\pi$ per unit degree of freedom. On the other hand, using the same criterion $(\kappa=+1)$ in both representations, which corresponds to Berry's phase of $2 \pi$ per unit degree of freedom, correctly reproduces the Hall conductivity of bilayer graphene [9]. For other discussions of Berry'a phases in mono- and bilayer graphene see, for instance, [22-24]. 


\section{Spinors in a constant electric field: pair production at $T=0$}

Now consider a graphene sample in the presence of a constant electrostatic field pointing toward the positive $O x$-axis, i.e. $F^{10}=F_{01}=E_{x}=E>0$. In this section, we will choose a gauge which leads to non-stationary sets of solutions. Throughout this section we will present a calculation, for the (2+1)-dimensional case, which is quite similar to that performed in [25] for the $(3+1)$-dimensional Minkowski spacetime. In particular, to solve the Dirac equation in the present $(2+1)$-dimensional massless case it is convenient to employ the following representation for the gamma matrices:

$$
\gamma^{0}=\sigma_{1}, \quad \gamma^{1}=\mathrm{i} \sigma_{2}, \quad \gamma^{2}=\mathrm{i} \sigma_{3} .
$$

After setting $\left(x^{0}, x^{1}, x^{2}\right)=(t, x, y)$, we get the massless Dirac operator in the gauge [25] $A^{\mu}=(0,-E t, 0)$, i.e.

$$
\not D=\mathrm{i} \partial_{t} \sigma_{1}+\left(\mathrm{i} \partial_{x}+e E t\right) \mathrm{i} \sigma_{2}-\partial_{y} \sigma_{3},
$$

which is explicitly time dependent. In order to obtain the solutions in this gauge, it is convenient to introduce the partial Fourier transforms

$$
\Psi(t, x, y)=\frac{1}{2 \pi} \int_{-\infty}^{\infty} \mathrm{d} p \int_{-\infty}^{\infty} \mathrm{d} k \mathrm{e}^{\mathrm{i} p x+\mathrm{i} k y} \widetilde{\Psi}(t, p, k),
$$

with $\mathbf{p}=(p, k)$ as well as the dimensionless quantities

$$
\xi \equiv(p-e E t) / \sqrt{e E}, \quad \lambda \equiv k^{2} / e E .
$$

If we write

$$
\Psi(t, x, y)=\left(\begin{array}{l}
\varphi(t, x, y) \\
\chi(t, x, y)
\end{array}\right), \quad \widetilde{\Psi}(t, p, k)=\left(\begin{array}{l}
\widetilde{\varphi}(t, p, k) \\
\widetilde{\chi}(t, p, k)
\end{array}\right),
$$

we obtain the coupled differential equations

$$
\left\{\begin{array}{l}
\mathrm{i} k \tilde{\varphi}+\sqrt{e E}\left(\mathrm{i} d_{\xi}+\xi\right) \tilde{\chi}=0, \\
\sqrt{e E}\left(-\mathrm{i} d_{\xi}+\xi\right) \widetilde{\varphi}+\mathrm{i} k \tilde{\chi}=0 .
\end{array}\right.
$$

Then, we can write

$$
\begin{aligned}
& \tilde{\chi}=(\mathrm{i} / \sqrt{\lambda})\left(-\mathrm{i} d_{\xi}+\xi\right) \tilde{\varphi}, \quad(\lambda \neq 0) \\
& \left(d_{\xi}^{2}+\xi^{2}+\lambda+\mathrm{i}\right) \widetilde{\varphi}=0 . \\
& \left(\mathrm{i} d_{\xi}+\xi\right) \tilde{\chi}=0, \quad\left(-\mathrm{i} d_{\xi}+\xi\right) \widetilde{\varphi}=0 \quad(\lambda=0) .
\end{aligned}
$$

The general solutions of equation (53) can be expressed in terms of two different sets of linearly independent solutions, involving parabolic cylinder functions [26]:

$$
\widetilde{\varphi}(\xi, \lambda)=A_{\text {in }} D_{-\mathrm{i} \lambda / 2}[+(1+\mathrm{i}) \xi]+B_{\mathrm{in}}^{*} D_{\mathrm{i} \lambda / 2-1}[+(1-\mathrm{i}) \xi],
$$

where $A_{\text {in }}$ and $B_{\text {in }}$ are complex constants. From the recursive relations

$$
\begin{aligned}
& \left(-\mathrm{i} d_{\xi}+\xi\right) D_{-\mathrm{i} \lambda / 2}[ \pm(1+\mathrm{i}) \xi]=\mp \frac{\lambda}{2}(1+\mathrm{i}) D_{-\mathrm{i} \lambda / 2-1}[ \pm(1+\mathrm{i}) \xi], \\
& \left(-\mathrm{i} d_{\xi}+\xi\right) D_{\mathrm{i} \lambda / 2-1}[ \pm(1-\mathrm{i}) \xi]= \pm(1+\mathrm{i}) D_{\mathrm{i} \lambda / 2}[ \pm(1-\mathrm{i}) \xi],
\end{aligned}
$$

we get the normalized spinors, which are solutions of the original massless Dirac equation and read as

$$
{ }_{-} \Psi_{\mathbf{p}}(t, \mathbf{x})=\frac{1}{4 \pi} \exp \left\{\mathrm{ip} \cdot \mathbf{x}-\frac{\pi \lambda}{8}\right\}\left(\begin{array}{c}
2 D_{-\mathrm{i} \lambda / 2}[(1+\mathrm{i}) \xi] \\
+(1-\mathrm{i}) \sqrt{\lambda} D_{-\mathrm{i} \lambda / 2-1}[(1+\mathrm{i}) \xi]
\end{array}\right),
$$


${ }_{+} \Psi_{\mathbf{p}}(t, \mathbf{x})=\frac{1}{4 \pi} \exp \left\{\mathrm{ip} \cdot \mathbf{x}-\frac{\pi \lambda}{8}\right\}\left(\begin{array}{c}-(1+\mathrm{i}) \sqrt{\lambda} D_{\mathrm{i} \lambda / 2-1}[(1-\mathrm{i}) \xi] \\ 2 D_{\mathrm{i} \lambda / 2}[(1-\mathrm{i}) \xi]\end{array}\right)$.

An alternative set of linearly independent solutions can be selected, that is,

$$
\widetilde{\varphi}(\xi, \lambda)=A_{\text {out }} D_{-\mathrm{i} \lambda / 2}[-(1+\mathrm{i}) \xi]+B_{\text {out }}^{*} D_{\mathrm{i} \lambda / 2-1}[-(1-\mathrm{i}) \xi] .
$$

Then, the recursive relations in equation (55) give

$$
\begin{aligned}
& { }^{+} \Psi_{\mathbf{p}}(t, \mathbf{x})=\frac{1}{4 \pi} \exp \left\{\mathrm{ip} \cdot \mathbf{x}-\frac{\pi \lambda}{8}\right\}\left(\begin{array}{c}
2 D_{-\mathrm{i} \lambda / 2}[-(1+\mathrm{i}) \xi] \\
-(1-\mathrm{i}) \sqrt{\lambda} D_{-\mathrm{i} \lambda / 2-1}[-(1+\mathrm{i}) \xi]
\end{array}\right), \\
& { }^{-} \Psi_{\mathbf{p}}(t, \mathbf{x})=\frac{1}{4 \pi} \exp \left\{\mathrm{ip} \cdot \mathbf{x}-\frac{\pi \lambda}{8}\right\}\left(\begin{array}{c}
+(1+\mathrm{i}) \sqrt{\lambda} D_{\mathrm{i} \lambda / 2-1}[-(1-\mathrm{i}) \xi] \\
2 D_{\mathrm{i} \lambda / 2}[-(1-\mathrm{i}) \xi]
\end{array}\right) .
\end{aligned}
$$

The above spinor solutions fulfill the orthonormality relations

$$
\begin{aligned}
& \left({ }_{ \pm} \Psi_{\mathbf{p}},{ }_{ \pm} \Psi_{\mathbf{q}}\right)=\int{ }_{ \pm} \Psi_{\mathbf{p}}^{\dagger}(t, \mathbf{x})_{ \pm} \Psi_{\mathbf{q}}(t, \mathbf{x}) \mathrm{d} \mathbf{x}=\delta(\mathbf{p}-\mathbf{q}), \\
& \left({ }^{ \pm} \Psi_{\mathbf{p}},{ }^{ \pm} \Psi_{\mathbf{q}}\right)=\int{ }^{ \pm} \Psi_{\mathbf{p}}^{\dagger}(t, \mathbf{x}){ }^{ \pm} \Psi_{\mathbf{q}}(t, \mathbf{x}) \mathrm{d} \mathbf{x}=\delta(\mathbf{p}-\mathbf{q}), \\
& \left({ } \Psi_{\mathbf{p}},{ }_{\mp} \Psi_{\mathbf{q}}\right)=0=\left({ }^{ \pm} \Psi_{\mathbf{p}},{ }^{\mp} \Psi_{\mathbf{q}}\right) .
\end{aligned}
$$

Note that for $\lambda=0=k$, owing to $D_{0}(z)=\exp \left\{-z^{2} / 4\right\}$, both pairs of solutions coincide and read as

$$
\begin{aligned}
& { }_{-} \Psi_{p}(t, \mathbf{x})={ }^{+} \Psi_{p}(t, \mathbf{x})=\frac{1}{2 \pi} \exp \left\{\mathrm{i} p x-\frac{\mathrm{i}}{2} \xi^{2}(t)\right\}\left(\begin{array}{l}
1 \\
0
\end{array}\right), \\
& +\Psi_{p}(t, \mathbf{x})={ }^{-} \Psi_{p}(t, \mathbf{x})=\frac{1}{2 \pi} \exp \left\{\mathrm{i} p x+\frac{\mathrm{i}}{2} \xi^{2}(t)\right\}\left(\begin{array}{l}
0 \\
1
\end{array}\right) .
\end{aligned}
$$

By looking at the asymptotic behavior of the parabolic cylinder functions, one finds

$$
\begin{array}{lll}
{ }_{-} \Psi_{\mathbf{p}}(t, \mathbf{x}) \sim \frac{1}{2 \pi}\left[2 \xi^{2}(t)\right]^{-\mathrm{i} \lambda / 4}\left(\begin{array}{l}
1 \\
0
\end{array}\right) \exp \left\{\mathrm{ip} \cdot \mathbf{x}-\frac{\mathrm{i}}{2} \xi^{2}(t)\right\} & (t \rightarrow-\infty) \\
{ }_{+} \Psi_{\mathbf{p}}(t, \mathbf{x}) \sim \frac{1}{2 \pi}\left[2 \xi^{2}(t)\right]^{\mathrm{i} \lambda / 4}\left(\begin{array}{l}
0 \\
1
\end{array}\right) \exp \left\{\mathrm{ip} \cdot \mathbf{x}+\frac{\mathrm{i}}{2} \xi^{2}(t)\right\} & (t \rightarrow-\infty) \\
{ }^{+} \Psi_{\mathbf{p}}(t, \mathbf{x}) \sim \frac{1}{2 \pi}\left[2 \xi^{2}(t)\right]^{-\mathrm{i} \lambda / 4}\left(\begin{array}{l}
1 \\
0
\end{array}\right) \exp \left\{\mathrm{ip} \cdot \mathbf{x}-\frac{\mathrm{i}}{2} \xi^{2}(t)\right\} & (t \rightarrow+\infty) \\
{ }^{-} \Psi_{\mathbf{p}}(t, \mathbf{x}) \sim \frac{1}{2 \pi}\left[2 \xi^{2}(t)\right]^{\mathrm{i} \lambda / 4}\left(\begin{array}{l}
0 \\
1
\end{array}\right) \exp \left\{\mathrm{ip} \cdot \mathbf{x}+\frac{\mathrm{i}}{2} \xi^{2}(t)\right\} & (t \rightarrow+\infty) .
\end{array}
$$

Hence, we understand ${ }_{+} \Psi_{\mathbf{p}}$ as the wavefunction of an incoming quasi-particle, while we associate the wavefunction $\Psi_{\mathbf{p}}$ with an incoming anti-quasi-particle. Conversely, we shall associate ${ }^{+} \Psi_{\mathbf{p}}$ and ${ }^{-} \Psi_{\mathbf{p}}$ with the corresponding outgoing wavefunctions [25].

The above sets of incoming and outgoing solutions are related throughout a Bogoliubovlike unitary transformation, that is,

$$
\left\{\begin{array}{l}
+\Psi_{\mathbf{p}}(x)=c_{1}^{+} \Psi_{\mathbf{p}}(x)+c_{2}^{-} \Psi_{\mathbf{p}}(x) \\
-\Psi_{\mathbf{p}}(x)=-c_{2}^{*+} \Psi_{\mathbf{p}}(x)+c_{1}^{*-} \Psi_{\mathbf{p}}(x),
\end{array} \quad\left|c_{1}\right|^{2}+\left|c_{2}\right|^{2}=1 .\right.
$$


We have

$$
\begin{aligned}
& \left({ }^{+} \Psi_{\mathbf{p}},{ }_{+} \Psi_{\mathbf{q}}\right)=N_{\lambda}^{*} \delta(\mathbf{p}-\mathbf{q}) \\
& \left({ }^{-} \Psi_{\mathbf{p}},{ }_{-} \Psi_{\mathbf{q}}\right)=N_{\lambda} \delta(\mathbf{p}-\mathbf{q}) \\
& \left({ }^{ \pm} \Psi_{\mathbf{p}},{ }_{\mp} \Psi_{\mathbf{q}}\right)=\exp \{-\pi \lambda / 2\} \delta(\mathbf{p}-\mathbf{q})
\end{aligned}
$$

where

$$
N_{\lambda}=\sqrt{\frac{\lambda}{\pi}} \Gamma\left(\frac{\mathrm{i} \lambda}{2}\right) \sinh \left(\frac{\pi \lambda}{2}\right) \exp \left\{-\frac{\pi \lambda}{4}+\frac{3 \pi \mathrm{i}}{4}\right\} .
$$

with

$$
\left|N_{\lambda}\right|^{2}=1-\exp \{-\pi \lambda\} .
$$

Then, using the above-listed orthonormality relations (61)-(63), we immediately find

$$
c_{1}=N_{\lambda}^{*}, \quad c_{2}=\exp \{-\pi \lambda / 2\}=c_{2}^{*} .
$$

As a consequence, in the transition to the quantum field theory, we can write the most general operator solutions of the Dirac equation as

$$
\begin{aligned}
& \Psi_{\text {in }}(x)=\int \mathrm{d} \mathbf{p}\left[a_{\text {in }}(\mathbf{p})_{+} \Psi_{\mathbf{p}}(x)+b_{\text {in }}^{\dagger}(\mathbf{p})_{-} \Psi_{\mathbf{p}}(x)\right], \\
& \Psi_{\text {out }}(x)=\int \mathrm{d} \mathbf{p}\left[a_{\text {out }}(\mathbf{p})^{+} \Psi_{\mathbf{p}}(x)+b_{\text {out }}^{\dagger}(\mathbf{p})^{-} \Psi_{\mathbf{p}}(x)\right],
\end{aligned}
$$

where each set of creation and destruction operators fulfills the standard canonical anticommutation relations. From the condition $\Psi_{\text {in }}(x)=\Psi_{\text {out }}(x)$, one has

$$
\begin{aligned}
& a_{\text {out }}(\mathbf{p})=c_{1} a_{\text {in }}(\mathbf{p})-c_{2} b_{\text {in }}^{\dagger}(\mathbf{p}), \\
& b_{\text {out }}^{\dagger}(\mathbf{p})=c_{2} a_{\text {in }}(\mathbf{p})+c_{1}^{*} b_{\text {in }}^{\dagger}(\mathbf{p}),
\end{aligned}
$$

so that

$$
\begin{aligned}
\langle 0 \mathbf{p} \text { out }| & =\langle 0 \mathbf{p} \text { in }| b_{\text {in }}(\mathbf{p}) b_{\text {out }}^{\dagger}\left(\mathbf{p}^{\prime}\right) \\
& =\langle 0 \mathbf{p} \text { in }| a_{\text {in }}(\mathbf{p}) a_{\text {out }}^{\dagger}\left(\mathbf{p}^{\prime}\right) \\
& =\langle 0 \mathbf{p} \text { in }|\left(c_{1}^{*} \delta\left(\mathbf{p}-\mathbf{p}^{\prime}\right)-c_{2} a_{\text {in }}\left(\mathbf{p}^{\prime}\right) b_{\text {in }}(\mathbf{p})\right) .
\end{aligned}
$$

Once we have the properly defined asymptotic in and out spinor states, which are explicitly gauge dependent, we can naturally define and calculate the gauge-invariant probabilities for the processes of pair creation and annihilation in the presence of the background electrostatic field. We start by obtaining the vacuum persistence amplitude at a given p. From equations (75) and (78), we find

$$
\begin{aligned}
\langle 0 \mathbf{p} \text { out }| 0 \mathbf{q} \text { in }\rangle & =c_{1}^{*} \delta(\mathbf{p}-\mathbf{q}) \\
& =\int \mathrm{d} \mathbf{x}^{-} \Psi_{\mathbf{p}}^{\dagger}(x)_{-} \Psi_{\mathbf{q}}(x)=N_{\lambda} \delta(\mathbf{p}-\mathbf{q}),
\end{aligned}
$$

with $N_{\lambda}$ given by equation (74).

In turn, the probability of vacuum decay at a given $\mathbf{p}$ is

$$
w_{\lambda}=1-\left|N_{\lambda}\right|^{2}
$$

or, more explicitly,

$$
w_{\lambda}=\exp \{-\pi \lambda\}, \quad \lambda=\frac{k^{2}}{e E} .
$$


It is clear that the latter one is also the probability of the inverse process, i.e. the annihilation in the state $\mathbf{p}$ with transfer of energy to the external field. As a matter of fact, we also have

$$
\int \mathrm{d} \mathbf{x}^{+} \Psi_{\mathbf{p}}^{\dagger}(t, \mathbf{x})_{-} \Psi_{\mathbf{q}}(t, \mathbf{x})=\delta(\mathbf{p}-\mathbf{q}) \exp \left\{-\frac{1}{2} \pi \lambda\right\} .
$$

The total vacuum persistence amplitude can be written, after recovering physical units, in the form (see the recent up-to-date review [27])

$$
\langle\text { out } 0| 0 \text { in }\rangle=\exp \left\{\frac{\mathrm{i}}{\hbar}\left[\operatorname{Re} \Gamma_{\text {eff }}(E)+\mathrm{i} \operatorname{Im} \Gamma_{\text {eff }}(E)\right]\right\} \text {, }
$$

and the corresponding probability is

$$
\mid\langle\text { out } 0| 0 \text { in }\rangle\left.\right|^{2}=\exp \left\{-\frac{2 \Omega \mathcal{T}}{\hbar} \operatorname{Im} \mathcal{L}_{\text {eff }}(E)\right\}
$$

where $\mathcal{L}_{\text {eff }}(E)$ is the effective Lagrange density in the presence of a background electrostatic field $E, \mathcal{T}$ is the total time and $\Omega$ denotes the area of the sample. It turns out that $\Gamma_{\text {eff }}(E)$ contains a real part that describes dispersive effects, such as the vacuum birefringence, as well as an imaginary part that concerns absorptive effects, such as vacuum decay. Now, according to the above derivation, the vacuum persistence probability can be obtained from

$$
\left.(\Omega \mathcal{T})^{-1} \ln \mid\langle\text { out } 0| 0 \text { in }\right\rangle\left.\right|^{2}=\frac{e E}{4 \pi^{2} \hbar^{2}} \int_{-\infty}^{\infty} \mathrm{d} k \ln \left(1-\exp \left\{-\frac{\pi k^{2} \tilde{c}}{e E \hbar}\right\}\right),
$$

where we have introduced the number of states between $\mathbf{p}$ and $\mathbf{p}+\mathrm{d} \mathbf{p}$, which is $\frac{\Omega}{4 \pi^{2} \hbar^{2}}$, and we have used that $\mathrm{d} p=e E \mathrm{~d} t$.

So, for the imaginary part of the effective action, we have, after taking the degeneracy $f$ into account,

$$
\begin{aligned}
\frac{2}{\hbar} \operatorname{Im} \mathcal{L}_{\text {eff }}(E) & =-f \frac{e E}{4 \pi^{2} \hbar^{2}} P \cdot V \cdot \int_{-\infty}^{\infty} \mathrm{d} k \ln \left(1-\exp \left\{-\frac{\pi k^{2} \tilde{c}}{e E \hbar}\right\}\right) \\
& =f \frac{(e E)^{\frac{3}{2}}}{4 \pi^{2} \hbar^{\frac{3}{2}} \tilde{c}^{\frac{1}{2}}} \sum_{n=1}^{\infty} \frac{1}{n^{3 / 2}}=f\left[\frac{\zeta\left(\frac{3}{2}\right)}{2 \pi}\right] \frac{e E}{2 \pi \hbar} \sqrt{\frac{e E}{\hbar \tilde{c}}},
\end{aligned}
$$

where $\tilde{c}$ is the speed of light for the two-dimensional graphene sample and $f$ is the number of fermion species or flavors, which is four for graphene, since the result is the same in both nonequivalent representations of the Clifford algebra. Moreover, the symbol P.V. stands for the Cauchy principal value prescription, i.e. P.V. $\int_{-\infty}^{\infty} \mathrm{d} k=2 \lim _{\epsilon \rightarrow 0} \int_{\epsilon}^{\infty} \mathrm{d} k$, which is the natural even prescription for the integrand around $k=0$. For previous, related results, see $[28,29]$.

Note that the vacuum persistence probability $\exp \left\{(-2 / \hbar) \Omega \mathcal{T} \operatorname{Im} \mathcal{L}_{\text {eff }}(E)\right\}$ is quite small, even at finite time and for a finite area, due to the massless character of the quasi-particles in graphene. So, the probability of the complementary event

$$
P=1-\exp \left(-\frac{2}{\hbar} \Omega \mathcal{T} \operatorname{Im} \mathcal{L}_{\text {eff }}(E)\right)
$$

is high and by no means approximatively given by the effective action as in the case of massive particles/antiparticles pairs. The vacuum persistence probability obtained by replacing (85) into this last equation coincides with the zero-mass limit of that reported in [30].

Some authors [31], starting with Schwinger [32], call this quantity the probability of pair creation. Others (see [30, 33] and references therein), following Nikishov [34], prefer to characterize the creation of pairs through the rate of pair production. Note that the rate of pair production was evaluated in the same number of dimensions, for the massive case, in [30]. In 
the zero mass limit, the result found in this last reference is given by the first term of the series in (85). This is exactly the result one would get after integrating (80) over all impulses, when counting the number of states between $\mathbf{p}$ and $\mathbf{p}+\mathrm{d} \mathbf{p}$ as before.

Apart from the way one characterizes vacuum decay, the important point is, indeed, that the probability of actually detecting vacuum decay effects is another appealing characteristic of graphene, at variance with the case of massive particles, in which vacuum decay is very hard to be unraveled (see [27]).

\section{Dirac field interacting with a constant electric background at finite temperature and density}

Now consider the problem of a graphene sample in the presence of a constant electric field pointing toward the positive $O x$-axis, i.e. $F^{10}=F_{01}=E_{x}=E>0$, at finite temperature and density.

As done in section 3, here we will employ path integral methods and zeta function regularization. In this section, at variance with what has been done in the previous one, we choose a gauge which leads to a stationary set of solutions.

To solve for the eigenvalues of the Dirac equation in the three-dimensional Euclidean space, with metric $(+,+,+)$, it is convenient to employ the following representation for the Euclidean gamma matrices:

$$
\bar{\gamma}_{1}=\sigma_{1}, \quad \bar{\gamma}_{2}=\sigma_{3}, \quad \bar{\gamma}_{3}=\sigma_{2} .
$$

After setting $\mathrm{i} x_{0}=\tau, x_{\mu}=\left(x_{1}, x_{2}, x_{3}\right)=(x, y, \tau)$ and taking into account that $\bar{A}_{\mu}=(0,0, \mathcal{E} x+\mathrm{i} v / e)(e>0)$ with $A_{0}=\mathrm{i} \bar{A}_{3}, E=\mathrm{i} \mathcal{E}$, we get the Euclidean Dirac operator

$$
\not D_{\text {Eucl }}=\left(\mathrm{i} \partial_{\tau}-e \mathcal{E} x-\mathrm{i} v\right) \sigma_{2}+\mathrm{i} \partial_{x} \sigma_{1}+\mathrm{i} \partial_{y} \sigma_{3} .
$$

In order to evaluate the effective action at finite temperature, one must impose antiperiodic boundary conditions on the eigenfunctions, as in section 3 . In this gauge, it is convenient to write the partial Fourier transform

$$
\Psi(x, y, \tau)=\frac{1}{\sqrt{ }(2 \pi \beta)} \sum_{\ell=-\infty}^{\infty} \exp \left\{\mathrm{i} \tau \omega_{\ell}\right\} \int_{-\infty}^{\infty} \mathrm{d} k \mathrm{e}^{\mathrm{i} k y} \widetilde{\Psi}_{\ell}(x, k),
$$

where, as before,

$$
\omega_{\ell}=\frac{2 \pi}{\beta}\left(\ell+\frac{1}{2}\right)
$$

denote the Matsubara angular frequencies.

Moreover, it turns out to be very convenient to introduce the dimensionless quantities

$$
\xi_{\ell} \equiv\left(\lambda_{\ell}+e \mathcal{E} x\right) / \sqrt{e \mathcal{E}}, \quad d_{\ell} \equiv \frac{\mathrm{d}}{\mathrm{d} \xi_{\ell}} \quad(\ell \in \mathbb{Z}),
$$

with $\lambda_{\ell}=\omega_{\ell}+\mathrm{i} v$, so that the eigenvalue equation reads as (note that, here, $\lambda$ represents the eigenvalues of the Euclidean Dirac operator and is different from $\lambda$ in the previous section)

$$
\sqrt{e \mathcal{E}}\left(\begin{array}{cc}
-k / \sqrt{e \mathcal{E}} & \mathrm{i} d_{\ell}+\mathrm{i} \xi_{\ell} \\
\mathrm{i} d_{\ell}-\mathrm{i} \xi_{\ell} & k / \sqrt{e \mathcal{E}}
\end{array}\right) \widetilde{\Psi}_{\ell}(x, k)=\lambda \widetilde{\Psi}_{\ell}(x, k) .
$$

If we write once again

$$
\widetilde{\Psi}_{\ell}(x, k) \equiv\left(\begin{array}{l}
\tilde{\varphi}_{\ell}(x, k) \\
\tilde{\chi}_{\ell}(x, k)
\end{array}\right)
$$


we are lead to the coupled differential equations

$$
\left\{\begin{array}{l}
\sqrt{e \mathcal{E}}\left(d_{\ell}+\xi_{\ell}\right) \tilde{\chi}_{\ell}+\mathrm{i}(\lambda+k) \tilde{\varphi}_{\ell}=0 \\
\sqrt{e \mathcal{E}}\left(d_{\ell}-\xi_{\ell}\right) \tilde{\varphi}_{\ell}+\mathrm{i}(\lambda-k) \tilde{\chi}_{\ell}=0 .
\end{array}\right.
$$

After solving these equations,

(i) When $\lambda=k$, we obtain the following infinite set of degenerate normalized improper eigenspinors, namely

$$
\begin{gathered}
\Psi_{0, k, \ell}(x, y, \tau)=\left(\frac{e \mathcal{E}}{\pi}\right)^{\frac{1}{4}} \exp \left\{\mathrm{i} \tau \omega_{\ell}+\mathrm{i} k y\right\}(2 \pi \beta)^{-\frac{1}{2}}\left(\begin{array}{c}
0 \\
\exp \left\{-\frac{1}{2} e \mathcal{E}\left(x+\lambda_{\ell} / e \mathcal{E}\right)^{2}\right\}
\end{array}\right), \\
\ell \in \mathbb{Z}, \quad k \in \mathbb{R},
\end{gathered}
$$

which fulfill

$\int_{0}^{\beta} \mathrm{d} \tau \int_{-\infty}^{\infty} \mathrm{d} x \int_{-\infty}^{\infty} \mathrm{d} y \Psi_{0, p, m}^{\dagger}(x, y, \tau) \Psi_{0, k, \ell}(x, y, \tau)=\delta_{\ell m} \delta(k-p)$.

Their degeneracy is

$$
\begin{aligned}
& \sum_{\ell=-\infty}^{\infty} \Psi_{0, k, \ell}^{\dagger}(x, y, \tau) \Psi_{0, k, \ell}(x, y, \tau) \\
& \quad=\frac{1}{2 \pi \beta} \sqrt{\frac{e \mathcal{E}}{\pi}} \sum_{\ell=-\infty}^{\infty} \exp \left\{-\frac{4 \pi^{2}}{e \mathcal{E} \beta^{2}}\left(\ell+\frac{1}{2}+z\right)^{2}\right\}=\frac{e \mathcal{E}}{4 \pi^{2}},
\end{aligned}
$$

where we have suitably introduced the rescaled and dimensionless complex coordinate $z=(e \mathcal{E} x+\mathrm{i} v) \frac{\beta}{2 \pi}$, while use has been made of the Euler-McLaurin summation formulasee for example equation (3.6.28) in [35]. Note that the $\ell$ th term of the series is an even function of $\ell$. Thus, all the odd derivatives vanish at $\ell=0$. Moreover, the $\ell$ th term and all its derivatives vanish at infinity. As a consequence we come to the result that the number of degenerate eigenspinors per unit Euclidean 'time' and unit $x$-length is provided by $\Delta_{\mathcal{E}}=e \mathcal{E} / 4 \pi^{2}$, the degeneracy factor being the same for all the remaining modes.

(ii) For $\lambda \neq k$, we can write

$$
\begin{aligned}
& \tilde{\chi}=\frac{\mathrm{i} \sqrt{e \mathcal{E}}}{\lambda-k}\left(d_{\ell}-\xi_{\ell}\right) \tilde{\varphi}, \\
& \left(d_{\ell}^{2}-\xi_{\ell}^{2}+\Lambda-1\right) \tilde{\varphi}=0, \quad \Lambda \equiv \frac{\lambda^{2}-k^{2}}{e \mathcal{E}}
\end{aligned}
$$

As in section 3 , the eigenvalue problem for each $\ell \in \mathbb{Z}$ is that of a linear harmonic oscillator, so that the spectrum takes the symmetric form

$$
\lambda_{k, n}= \pm \sqrt{k^{2}+2 e \mathcal{E} n}, \quad n=1, \ldots, \infty, \quad k \in \mathbb{R} \quad(\forall \ell \in \mathbb{Z}) .
$$

The somewhat surprising conclusion we can draw from the above analysis is that neither the eigenvalues nor the degeneracy depend at all upon the temperature and/or the chemical potential. Hence, once we have at hand the spectrum of the Euclidean Dirac operator and its overall degeneracy, which is the same for all eigenvalues, we can turn to the evaluation of the Euclidean effective action, within the zeta function approach. In this case, we have

$\zeta\left(s, \not D \ell_{y}\right)=\Delta_{\mathcal{E}} \beta \ell_{x} \ell_{y} \int_{-\infty}^{\infty} \mathrm{d} k\left[\left(\ell_{y} k\right)^{-s}+\frac{1+\mathrm{e}^{\mp \pi \mathrm{i} s}}{\left(2 e \mathcal{E} \ell_{y}{ }^{2}\right)^{s / 2}} \sum_{n=1}^{\infty}\left(n+\frac{k^{2}}{2 e \mathcal{E}}\right)^{-s / 2}\right]$. 
Here, the length scale $\ell_{y}$ has been introduced to render the second argument in the zeta function dimensionless.

Now, equation (98) can be rewritten as

$$
\begin{aligned}
\zeta\left(s, \not D \ell_{y}\right)= & \Delta_{\mathcal{E}} \Omega \beta\left\{\int_{-\infty}^{\infty} \mathrm{d} k\left(\ell_{y} k\right)^{-s}\right. \\
& \left.+\frac{1+\mathrm{e}^{\mp \pi \mathrm{i} s}}{\Gamma(s / 2)\left(2 e \mathcal{E} \ell_{y}^{2}\right)^{s / 2}} \int_{-\infty}^{\infty} \mathrm{d} k \int_{0}^{\infty} \mathrm{d} t t^{s / 2-1} \sum_{n=1}^{\infty} \mathrm{e}^{-t n-t k^{2} / 2 e \mathcal{E}}\right\} .
\end{aligned}
$$

For Re $s>1$ the two integrals in the second line can be interchanged and, if the integral in the first line is understood in terms of the Cauchy principal value prescription, according to our comment in section 4 , we get

$$
\begin{aligned}
\zeta\left(s, \not D \ell_{y}\right)= & \Delta_{\mathcal{E}} \Omega \beta\left(1+\mathrm{e}^{\mp \pi \mathrm{is}}\right) \ell_{y}{ }^{-s} \\
& \times\left[\lim _{\epsilon \rightarrow 0} \frac{(\epsilon)^{1-s}}{1-s}+\frac{(s / 2) \sqrt{\pi}}{\Gamma(1+s / 2)}(2 e \mathcal{E})^{\frac{1-s}{2}} \zeta\left(\frac{s-1}{2}\right) \Gamma\left(\frac{s-1}{2}\right)\right] .
\end{aligned}
$$

We finally obtain the Euclidean effective action per unit volume in the form (note, however, that the contribution coming from the first term was defined here as $\left.-\lim _{\epsilon \rightarrow 0} \int_{\epsilon}^{\infty} \mathrm{d} k \frac{\mathrm{d}}{\mathrm{d} s}\right\rfloor_{s=0}\left(\ell_{y} k\right)^{-s}$, the integrand being evaluated at $\operatorname{Re} s>1$ and then extended to $s=0$ before taking the principal value limit)

$$
\begin{aligned}
\frac{1}{\Omega \beta}\left[-\frac{\mathrm{d}}{\mathrm{d} s} \zeta(s, \not D \beta)\right]_{s=0} & =-\Delta_{\mathcal{E}} \Gamma\left(-\frac{1}{2}\right) \zeta\left(-\frac{1}{2}\right) \sqrt{2 \pi \mathcal{E}} \\
& =-2 \sqrt{2} \pi \Delta_{\mathcal{E}} \zeta\left(-\frac{1}{2}\right) \sqrt{e \mathcal{E}} .
\end{aligned}
$$

where (see [26])

$$
2 \sqrt{2} \zeta\left(-\frac{1}{2}\right)=\frac{1}{\pi} \sum_{n=1}^{\infty} n^{-3 / 2} \sin \left(-\frac{\pi}{4}\right)=-\frac{\zeta\left(\frac{3}{2}\right)}{\pi \sqrt{ } 2} .
$$

As a consequence, we can recast the Euclidean effective Lagrangian as

$$
\mathcal{L}_{\text {eff }}^{\mathrm{E}}=\frac{\zeta\left(\frac{3}{2}\right)}{4 \pi^{2} \sqrt{ } 2}(e \mathcal{E})^{3 / 2}
$$

so that, after turning back to the (2+1)-dimensional Minkowski spacetime by means of the replacement $\mathcal{E}=-\mathrm{i} E$, we obtain the effective Lagrange density

$$
\mathcal{L}_{\text {eff }}^{\mathrm{M}}(E)=\frac{\zeta\left(\frac{3}{2}\right)}{8 \pi^{2}}(e E)^{3 / 2}[(1+\mathrm{i})] .
$$

It follows that, as in equation (83), we end up with the correspondence

$$
\langle\text { out } 0| 0 \text { in }\rangle=\exp \left\{\mathrm{i} \Omega \mathcal{T} \mathcal{L}_{\text {eff }}^{\mathrm{M}}(E)\right\}=\exp \left\{\mathrm{i} \Omega \mathcal{T} \frac{\zeta\left(\frac{3}{2}\right)}{8 \pi^{2}}(e E)^{3 / 2}(1+\mathrm{i})\right\},
$$

which leads, after recovering physical units, to equation (85), i.e. the (2+1)-dimensional counterpart of the celebrated Schwinger formula [32]

$$
\operatorname{Im} \mathcal{L}_{\text {eff }}^{\mathrm{M}}(E)=f \frac{\zeta\left(\frac{3}{2}\right)(e E)^{\frac{3}{2}}}{8 \pi^{2} \hbar \sqrt{ } \hbar \tilde{c}}
$$

where $\tilde{c}$ is the speed of light for the two-dimensional graphene sample and $f$ is the number of fermion species or flavors, which is four for graphene, in perfect agreement with our previous result (85). Moreover we find that in the (2+1)-dimensional case the following remarkable equalities hold true, namely

$$
\operatorname{Re} \mathcal{L}_{\text {eff }}^{\mathrm{M}}(E)=\operatorname{Im} \mathcal{L}_{\text {eff }}^{\mathrm{M}}(E)=-\mathcal{L}_{\text {eff }}^{\mathrm{E}}(\mathcal{E})
$$




\section{Minimal quantum conductivity of graphene from planar QED}

Of particular interest in the presence of a constant electric background is the mean current density or, equivalently, the minimal conductivity of graphene. In order to evaluate the mean value of such quantum current density, we go back to our calculation in the previous section, this time introducing a Lagrange multiplier $\alpha$ through the non-trivial replacement $k \rightarrow(k+\mathrm{i} \alpha)$. The derivative of the effective action with respect to the external parameter $\alpha$ will then give us the required mean value of the quantum current density. Actually, we can say that the control parameter $\alpha$ plays a role very similar to that played by the chemical potential in the evaluation of the particle number or charge density.

If we consider the manifold to be compact in the $y$ direction, all we have to do is to turn back to equation (100), where we have, after a shift in the transverse momentum integration variable $k$,

$$
\begin{aligned}
\zeta\left(s, \not D \ell_{y} ; \alpha\right)= & \Delta_{\mathcal{E}} \Omega \beta \ell_{y}{ }^{-s}\left\{\int_{-\infty}^{\infty} \mathrm{d} k(k+\mathrm{i} \alpha)^{-s}\right. \\
& \left.+\frac{1+\mathrm{e}^{\mp \pi i s}}{\Gamma(s / 2)(2 e \mathcal{E})^{s / 2}} \int_{-\infty}^{\infty} \mathrm{d} k \int_{0}^{\infty} \mathrm{d} t t^{s / 2-1} \sum_{n=1}^{\infty} \mathrm{e}^{-t n-t(k+\mathrm{i} \alpha)^{2} / 2 e \mathcal{E}}\right\} .
\end{aligned}
$$

Now, the shift in the second term is irrelevant, since the Gaussian function is an entire function. Then, the $\alpha$-dependence is solely due to the first term and, consequently, the only contribution to the minimal quantum current density will arise from the quantity

$$
\begin{aligned}
Z_{\mathcal{E}}(s ; \alpha) & =\Delta_{\mathcal{E}} \Omega \beta \ell_{y}{ }^{-s} \int_{-\infty}^{\infty} \mathrm{d} k(k+\mathrm{i} \alpha)^{-s} \\
& =\Delta_{\mathcal{E}} \Omega \beta \ell_{y}{ }^{-s} \int_{0}^{\infty} \mathrm{d} k\left[(k+\mathrm{i} \alpha)^{-s}+(-1)^{-s}(k-\mathrm{i} \alpha)^{-s}\right] .
\end{aligned}
$$

At variance with the case of the previous section $(\alpha=0)$, the zeta regularization of this term is now well defined, the only ambiguity being the selection of the phase, much as in the calculation of the charge when the sample is subjected to a magnetic field. As in that case, one can make two selections of the phase. Indeed,

$$
Z_{\mathcal{E}}(s ; \alpha)=\Delta_{\mathcal{E}} \Omega \beta \ell_{y}{ }^{-s} \int_{0}^{\infty} \mathrm{d} k\left[(k+\mathrm{i} \alpha)^{-s}+\mathrm{e}^{-\pi \mathrm{i} s \kappa \operatorname{sign}(\alpha)}(k-\mathrm{i} \alpha)^{-s}\right],
$$

where, as before, $\kappa=-1$ represents the 'usual' selection of phase, whilst $\kappa=1$ the opposite 'unusual' one. Thus, for $\operatorname{Re} s>1$, we get

$$
Z_{\mathcal{E}}(s ; \alpha)=-\Delta_{\mathcal{E}} \Omega \frac{(\mathrm{i} \alpha \beta)^{1-s}}{1-s}\left[1-\mathrm{e}^{-2 \pi \mathrm{i} s \kappa \operatorname{sign}(\alpha)}\right] .
$$

Performing the $s$-derivative and evaluating at $s=0$, we get for the $\alpha$-dependent part of the Euclidean effective action

$$
\Gamma_{\mathrm{eff}}^{\mathrm{E}}(\alpha)=-2 \pi \Delta_{\mathcal{E}} \Omega \beta \kappa|\alpha|=-\frac{e \mathcal{E}}{2 \pi} \beta \Omega \kappa|\alpha| .
$$

To the aim of recovering the minimal quantum current density it is necessary, first, to go back to the (2+1)-dimensional Minkowski spacetime, i.e.

$$
\Gamma_{\mathrm{eff}}^{\mathrm{M}}(\alpha)=\frac{e E}{2 \pi} \mathcal{T} \Omega \kappa|\alpha| .
$$

Now, it turns out that performing the derivative with respect to $\alpha$, dividing by $\Omega \mathcal{T}$ and multiplying by the elementary charge, $-e$, we have

$$
\left\langle J_{\min }\right\rangle=-\frac{e^{2} E}{2 \pi} \kappa \operatorname{sign}(\alpha)
$$


Note that, in the other representation, the result is the same if the same criterion is chosen to define the phase of the determinant. Thus, summing up the contributions from both representations, in contrast to the case of the Hall conductivity, with the same criterion, multiplying by the two spins (flavors) and recovering the physical units, we find for graphene

$$
\left\langle J_{\min }\right\rangle=-\frac{4 e^{2}}{h} E \kappa \operatorname{sign}(\alpha) .
$$

The quantization of the minimal quantum conductivity

$$
\sigma_{\min }=-\frac{4 e^{2}}{h} \kappa \operatorname{sign}(\alpha)
$$

in terms of the quantum unit of conductivity makes this prediction entirely different from the results obtained, for instance, through the Kubo formula $[15,16]$, where an extra factor of $\pi$ in the denominator appears. Moreover, for $\kappa=-1$ and positive $\alpha$ one obtains exactly the result in [8]. It is interesting to note that a similar experimental value is also found for bilayer graphene [24].

As already stressed, this result is independent of both the temperature and the chemical potential.

\section{Conclusions}

In this paper, we have carefully analyzed the response of a graphene sample under the influence of homogeneous electric and magnetic fields. Our present investigation has its roots in the well-established quantum field theoretic model of a free massless Dirac spinor in two space and one time dimensions, i.e. the massless planar spinor quantum electrodynamics. In spite of its simplicity, this quantum field theory does exhibit remarkable features. As a matter of fact, in the presence of a uniform magnetic field at finite temperature and density, a nice connection has been established, in this paper, among the phase prescription of the effective action, the large gauge transformation invariance, Berry's phases and Chern-Simons topological terms, besides the different forms for the Hall conductivity we had already elucidated in our previous paper [9].

Moreover, in the presence of a constant electrostatic field, a derivation of the imaginary part of the effective Lagrangian at zero temperature has been presented. Our result coincides with the zero mass limit of that obtained, for instance, in [30].

Interestingly enough, at finite temperature, the imaginary part of the effective Lagrangian turns out to be independent of the temperature and density and coincides with the massless planar limit of the celebrated Schwinger formula [32]. The corresponding probability of vacuum decay, for a realistic graphene sample, can be very high for laboratory fields, at variance with the common case of massive charged electron positron pairs. In the case of $3+1$ dimensions, such independence was shown to hold, to the order of one loop, in [2]. Our result in $2+1$ is stronger: indeed, our calculations show that the whole effective Lagrangian is independent of the temperature and of the chemical potential, within the zeta function regularization scheme.

Furthermore, our treatment, based on the zeta function regularization technique, allowed us to obtain the (finite) average value for the minimal quantum current density and, thus, a minimal quantum conductivity for graphene, which, at variance with most theoretical results $[15,16]$, agrees, for a particular selection of the phase of the determinant in both irreducible representations, with that exhibited in [8]. The corresponding current is topological in origin, as is the behavior of the Hall conductivity (for general studies of topological currents in $2+1$ 
QED see, for example, [29, 36]). We hope the present paper will be helpful in clarifying the so-called mystery of the missing pi [8] or its absence thereof [37].

\section{Acknowledgments}

This work was partially supported by Universidad Nacional de La Plata (Proyecto 11/X492) and CONICET (PIP 6160). PG and RS would like to thank the Istituto Nazionale di Fisica Nucleare for grant I.S. PI13. EMS thanks S Gavrilov, T Cohen and N Protasov for useful comments and discussions.

\section{Appendix. Parabolic cylinder functions}

The parabolic cylinder functions, of the special form we are interested in the present context, can be defined, e.g., by the integral representation (9.241) 1. p 1092 of [26]:

$D_{-\mathrm{i} \lambda / 2}[ \pm(1+\mathrm{i}) \xi]=\frac{1}{\sqrt{ } \pi} 2^{-\mathrm{i} \lambda / 2+1 / 2} \mathrm{e}^{-\pi \lambda / 4} \mathrm{e}^{\mathrm{i} \xi^{2} / 2} \int_{-\infty}^{\infty} x^{-\mathrm{i} \lambda / 2} \mathrm{e}^{-2 x^{2} \pm 2 \mathrm{i} x(1+\mathrm{i}) \xi} \mathrm{d} x$,

where $\lambda>0, \xi \in \mathbb{R}, \arg x^{-\mathrm{i} \lambda / 2}=\lambda / 2$ for $x<0$, so that

$D_{-\mathrm{i} \lambda / 2}^{*}[ \pm(1+\mathrm{i}) \xi]=\frac{1}{\sqrt{ } \pi} 2^{\mathrm{i} \lambda / 2+1 / 2} \mathrm{e}^{-\pi \lambda / 4} \mathrm{e}^{-\mathrm{i} \xi^{2} / 2} \int_{-\infty}^{\infty} x^{\mathrm{i} \lambda / 2} \mathrm{e}^{-2 x^{2} \mp 2 \mathrm{i} x(1-\mathrm{i}) \xi} \mathrm{d} x$.

After the change of variable $x \longmapsto-x$

$D_{-\mathrm{i} \lambda / 2}^{*}[ \pm(1+\mathrm{i}) \xi]=\frac{1}{\sqrt{ } \pi} 2^{\mathrm{i} \lambda / 2+1 / 2} \mathrm{e}^{\pi \lambda / 4} \mathrm{e}^{-\mathrm{i} \xi^{2} / 2} \int_{-\infty}^{\infty} x^{\mathrm{i} \lambda / 2} \mathrm{e}^{-2 x^{2} \pm 2 \mathrm{i} x(1-\mathrm{i}) \xi} \mathrm{d} x$,

we eventually come to the conjugation property

$$
D_{-\mathrm{i} \lambda / 2}^{*}[ \pm(1+\mathrm{i}) \xi]=D_{\mathrm{i} \lambda / 2}[ \pm(1-\mathrm{i}) \xi],
$$

as naïvely expected. The following special values appear in our calculations:

$$
\begin{aligned}
& D_{ \pm \mathrm{i} \lambda / 2}(0)=\pi^{-1 / 2} 2^{ \pm \mathrm{i} \lambda / 4} \Gamma\left(\frac{1}{2} \pm \frac{\mathrm{i} \lambda}{4}\right) \cosh \frac{\pi \lambda}{4} \\
& D_{ \pm \mathrm{i} \lambda / 2-1}(0)= \pm \mathrm{i} \pi^{-1 / 2} 2^{ \pm \mathrm{i} \lambda / 4-1 / 2} \Gamma\left( \pm \frac{\mathrm{i} \lambda}{4}\right) \sinh \frac{\pi \lambda}{4} \\
& \pm \frac{\lambda}{2}\left|D_{ \pm \mathrm{i} \lambda / 2-1}(0)\right|^{2}= \pm \sinh \frac{\pi \lambda}{4} \\
& \left|D_{ \pm \mathrm{i} \lambda / 2}(0)\right|^{2}=\cosh \frac{\pi \lambda}{4} .
\end{aligned}
$$

The parabolic cylinder functions fulfill the recursion formulae

$$
\begin{aligned}
& \frac{\mathrm{d}}{\mathrm{d} z} D_{v}(z)=-\frac{1}{2} z D_{v}(z)+v D_{v-1}(z) \\
& \frac{\mathrm{d}}{\mathrm{d} z} D_{v}(z)=\frac{1}{2} z D_{v}(z)-D_{v+1}(z)
\end{aligned}
$$

Consider the combination

$D_{+} \equiv D_{-\mathrm{i} \lambda / 2}[(1+\mathrm{i}) \xi] D_{\mathrm{i} \lambda / 2}[(1-\mathrm{i}) \xi]+\frac{\lambda}{2} D_{-\mathrm{i} \lambda / 2-1}[(1+\mathrm{i}) \xi] D_{\mathrm{i} \lambda / 2-1}[(1-\mathrm{i}) \xi]$. 
From the recursion formulae, we get

$2 \frac{\mathrm{d}}{\mathrm{d} \xi} D_{-\mathrm{i} \lambda / 2}[(1+\mathrm{i}) \xi] D_{\mathrm{i} \lambda / 2}[(1-\mathrm{i}) \xi]=\lambda(1+\mathrm{i}) D_{-\mathrm{i} \lambda / 2}[(1+\mathrm{i}) \xi] D_{\mathrm{i} \lambda / 2-1}[(1-\mathrm{i}) \xi]+$ c.c.

$\lambda \frac{\mathrm{d}}{\mathrm{d} \xi} D_{-\mathrm{i} \lambda / 2-1}[(1+\mathrm{i}) \xi] D_{\mathrm{i} \lambda / 2-1}[(1-\mathrm{i}) \xi]=-\lambda(1+\mathrm{i}) D_{-\mathrm{i} \lambda / 2}[(1+\mathrm{i}) \xi] D_{\mathrm{i} \lambda / 2-1}[(1-\mathrm{i}) \xi]+\mathrm{c} . \mathrm{c}$.

so that the above combination $D_{+}$does not depend upon $\xi$ and from the conjugation property (A.4) we can write

$$
D_{+}=\left|D_{-\mathrm{i} \lambda / 2}(0)\right|^{2}+\frac{\lambda}{2}\left|D_{-\mathrm{i} \lambda / 2-1}(0)\right|^{2}=\exp \{\pi \lambda / 4\}
$$

Let us now consider the further combination

$D_{-} \equiv D_{-\mathrm{i} \lambda / 2}[(1+\mathrm{i}) \xi] D_{\mathrm{i} \lambda / 2}[-(1-\mathrm{i}) \xi]-\frac{\lambda}{2} D_{-\mathrm{i} \lambda / 2-1}[(1+\mathrm{i}) \xi] D_{\mathrm{i} \lambda / 2-1}[-(1-\mathrm{i}) \xi]$

From the recursion formulae, we get

$$
\begin{gathered}
\begin{array}{c}
2 \frac{\mathrm{d}}{\mathrm{d} \xi}\left\{D_{\mathrm{i} \lambda / 2}[(\mathrm{i}-1) \xi] D_{-\mathrm{i} \lambda / 2}[(\mathrm{i}+1) \xi]\right\}=\lambda(1-\mathrm{i}) D_{\mathrm{i} \lambda / 2}[(\mathrm{i}-1) \xi] D_{-\mathrm{i} \lambda / 2-1}[(\mathrm{i}+1) \xi] \\
-\lambda(1+\mathrm{i}) D_{-\mathrm{i} \lambda / 2}[(\mathrm{i}+1) \xi] D_{\mathrm{i} \lambda / 2-1}[(\mathrm{i}-1) \xi]
\end{array} \\
\begin{array}{c}
\lambda \frac{\mathrm{d}}{\mathrm{d} \xi}\left\{D_{-\mathrm{i} \lambda / 2-1}[(\mathrm{i}+1) \xi] D_{\mathrm{i} \lambda / 2-1}[(\mathrm{i}-1) \xi]\right\}=\lambda(1-\mathrm{i}) D_{\mathrm{i} \lambda / 2}[(\mathrm{i}-1) \xi] D_{-\mathrm{i} \lambda / 2-1}[(\mathrm{i}+1) \xi] \\
-\lambda(1+\mathrm{i}) D_{-\mathrm{i} \lambda / 2}[(\mathrm{i}+1) \xi] D_{\mathrm{i} \lambda / 2-1}[(\mathrm{i}-1) \xi]
\end{array}
\end{gathered}
$$

which also leads to the conclusion that the quantity $D_{-}$is independent of $\xi$, and yields

$$
D_{-}=\left|D_{-\mathrm{i} \lambda / 2}(0)\right|^{2}-\frac{\lambda}{2}\left|D_{-\mathrm{i} \lambda / 2-1}(0)\right|^{2}=\exp \{-\pi \lambda / 4\}
$$

The above important properties of the parabolic cylinder functions can be summarized in the remarkable formula

$$
D_{ \pm}=\left|D_{\mathrm{i} \lambda / 2}(0)\right|^{2} \pm \frac{\lambda}{2}\left|D_{\mathrm{i} \lambda / 2-1}(0)\right|^{2}=\exp \{ \pm \pi \lambda / 4\}
$$

Consider the second-order differential equations

$$
\left(\frac{\mathrm{d}^{2}}{\mathrm{~d} \xi^{2}}+\xi^{2}+\lambda \pm \mathrm{i}\right) f_{ \pm}(\xi, \lambda)=0 .
$$

Two pairs of linearly independent solutions for the upper sign equation are

$$
\begin{aligned}
& f_{+}^{(1)}( \pm \xi, \lambda)=D_{-\mathrm{i} \lambda / 2}\left( \pm \xi \sqrt{2} \mathrm{e}^{\pi \mathrm{i} / 4}\right) \\
& f_{+}^{(2)}( \pm \xi, \lambda)=D_{\mathrm{i} \lambda / 2-1}\left( \pm \xi \sqrt{2} \mathrm{e}^{-\pi \mathrm{i} / 4}\right)
\end{aligned}
$$

while two couples of linearly independent solutions for the lower sign equation are

$$
\begin{aligned}
& f_{-}^{(1)}( \pm \xi, \lambda)=D_{-\mathrm{i} \lambda / 2-1}\left( \pm \xi \sqrt{2} \mathrm{e}^{\pi \mathrm{i} / 4}\right) \\
& f_{-}^{(2)}( \pm \xi, \lambda)=D_{\mathrm{i} \lambda / 2}\left( \pm \xi \sqrt{2} \mathrm{e}^{-\pi \mathrm{i} / 4}\right)
\end{aligned}
$$

To the aim of verifying linear independence, we have to compute the Wronskian. Let us first calculate derivatives by means of the recursion formulae (A.9) and (A.10) that yield $\frac{\mathrm{d}}{\mathrm{d} \xi} D_{-\mathrm{i} \lambda / 2}\left( \pm \xi \sqrt{2} \mathrm{e}^{\pi \mathrm{i} / 4}\right)=-\mathrm{i} \xi D_{-\mathrm{i} \lambda / 2}\left( \pm \xi \sqrt{2} \mathrm{e}^{\pi \mathrm{i} / 4}\right) \mp \frac{\lambda}{\sqrt{ } 2} \mathrm{e}^{3 \pi \mathrm{i} / 4} D_{-\mathrm{i} \lambda / 2-1}\left( \pm \xi \sqrt{2} \mathrm{e}^{\pi \mathrm{i} / 4}\right)$ 
and thereby

$$
W\left[f_{+}^{(1)}(\xi, \lambda), f_{+}^{(1)}(-\xi, \lambda)\right]=\frac{1+\mathrm{i}}{\sqrt{ } \pi} \Gamma\left(-\frac{\mathrm{i} \lambda}{2}\right) \sinh \left(\frac{\pi \lambda}{2}\right) .
$$

On the other hand, we readily find

$$
W\left[f_{+}^{(1)}( \pm \xi, \lambda), f_{+}^{(2)}( \pm \xi, \lambda)\right]=\mp(1-i) \exp \{\pi \lambda / 4\}
$$

and analogous relationships for the other solutions.

In order to understand the physical meaning of the solutions of the wave field equations, we have to analyze the leading asymptotic behavior of the parabolic cylinder functions. Then from equation (9.246) 1. p 1093 of [26], we have

$$
\left.\begin{array}{c}
D_{-\mathrm{i} \lambda / 2}\left(\xi \sqrt{2} \mathrm{e}^{\pi \mathrm{i} / 4}\right) \sim\left(2 \xi^{2}\right)^{-\mathrm{i} \lambda / 4} \mathrm{e}^{\pi \lambda / 8} \exp \left\{-\mathrm{i} \xi^{2} / 2\right\} \\
D_{-\mathrm{i} \lambda / 2-1}\left(\xi \sqrt{2} \mathrm{e}^{\pi \mathrm{i} / 4}\right) \sim O\left(\xi^{-1}\right)
\end{array}\right\} \quad(\xi \gg \lambda>0) .
$$

If instead $\xi \ll-\lambda$, we have either $\xi \mathrm{e}^{\pi \mathrm{i} / 4}=|\xi| \mathrm{e}^{5 \pi \mathrm{i} / 4}$ or else $\xi \mathrm{e}^{\pi \mathrm{i} / 4}=|\xi| \mathrm{e}^{-3 \pi \mathrm{i} / 4}$. Now, for $\arg \left(\xi \mathrm{e}^{\pi \mathrm{i} / 4}\right)=5 \pi \mathrm{i} / 4$, no reliable asymptotic expansion is available, so that from equation (9.246) 3., p 1094 of [26] we obtain the bona fide leading behavior for $\xi \ll-\lambda<0$, namely

$$
\begin{array}{r}
D_{-\mathrm{i} \lambda / 2-1}\left(\xi \sqrt{2} \mathrm{e}^{\pi \mathrm{i} / 4}\right)=D_{-\mathrm{i} \lambda / 2-1}\left(|\xi| \sqrt{2} \mathrm{e}^{-3 \pi \mathrm{i} / 4}\right) \\
\sim \frac{\sqrt{2 \pi}}{\Gamma(1+\mathrm{i} \lambda / 2)}\left(2 \xi^{2}\right)^{\mathrm{i} \lambda / 4} \exp \left\{-\frac{\pi \lambda}{8}+\frac{\mathrm{i} \xi^{2}}{2}\right\}, \\
D_{-\mathrm{i} \lambda / 2}\left(|\xi| \sqrt{2} \mathrm{e}^{-3 \pi \mathrm{i} / 4}\right) \sim\left(2 \xi^{2}\right)^{-\mathrm{i} \lambda / 4} \exp \left\{-\frac{3 \pi \lambda}{8}-\frac{\mathrm{i} \xi^{2}}{2}\right\} .
\end{array}
$$

Of course, the situation becomes exactly time-reversed for the two other linearly independent solutions: namely, for $\xi \gg \lambda>0$ we find

$$
\begin{gathered}
D_{-\mathrm{i} \lambda / 2-1}\left(-\xi \sqrt{2} \mathrm{e}^{\pi \mathrm{i} / 4}\right)=D_{-\mathrm{i} \lambda / 2-1}\left(\xi \sqrt{2} \mathrm{e}^{-3 \pi \mathrm{i} / 4}\right) \\
\sim \frac{\sqrt{2 \pi}}{\Gamma(1+\mathrm{i} \lambda / 2)}\left(2 \xi^{2}\right)^{\mathrm{i} \lambda / 4} \exp \left\{-\frac{\pi \lambda}{8}+\frac{\mathrm{i} \xi^{2}}{2}\right\}, \\
D_{-\mathrm{i} \lambda / 2}\left(-\xi \sqrt{2} \mathrm{e}^{\pi \mathrm{i} / 4}\right)=D_{-\mathrm{i} \lambda / 2}\left(\xi \sqrt{2} \mathrm{e}^{-3 \pi \mathrm{i} / 4}\right) \\
\sim\left(2 \xi^{2}\right)^{-\mathrm{i} \lambda / 4} \exp \left\{-\frac{3 \pi \lambda}{8}-\frac{\mathrm{i} \xi^{2}}{2}\right\}
\end{gathered}
$$

whereas for $\xi \ll-\lambda<0$ we obtain

$$
\left.\begin{array}{c}
D_{-\mathrm{i} \lambda / 2}\left(|\xi| \sqrt{2} \mathrm{e}^{\pi \mathrm{i} / 4}\right) \sim\left(2 \xi^{2}\right)^{-\mathrm{i} \lambda / 4} \mathrm{e}^{\pi \lambda / 8} \exp \left\{-\mathrm{i} \xi^{2} / 2\right\} \\
D_{-\mathrm{i} \lambda / 2-1}\left(|\xi| \sqrt{2} \mathrm{e}^{\pi \mathrm{i} / 4}\right) \sim O\left(\xi^{-1}\right)
\end{array}\right\} \quad(\xi \ll-\lambda<0)
$$

For a given particle momentum $p_{x}=p$, we shall associate the stationary asymptotic phase

$$
p x-\frac{1}{2} \xi^{2}(t)=p x-\frac{1}{2} e E t^{2}+p t-\frac{p^{2}}{2 e E}
$$

with the positive frequency solutions $\xi^{2}(t)$ which describe a particle, i.e. an electron of momentum $p$, while the stationary asymptotic phase

$$
p x+\frac{1}{2} \xi^{2}(t)=p x+\frac{1}{2} e E t^{2}-p t+\frac{p^{2}}{2 e E}
$$

will describe an antiparticle, i.e. a positron of momentum $-p$. 


\section{References}

[1] Dunne G V 1999 Topological Aspects of Low Dimensional Systems (Berlin: Springer)

[2] Dittrich W and Gies H 2000 Probing the Vacuum: Perturbative Effective Action Approach in Quantum Electrodynamics and its Application (Springer Tracts in Modern Physics vol 166) (Berlin, Heidelberg: Springer)

[3] Raya A and Reyes E 2008 J. Phys. A: Math. Theor. 41355401

[4] Semenoff G W 1984 Phys. Rev. Lett. 532499

[5] DiVincenzo D P and Mele E J 1984 Phys. Rev. B 291685

[6] Novoselov K S, Geim A K, Morozov S V, Jiang D, Zhang Y, Dubonos S V, Grigorieva I V and Firsov A A 2004 Science 306666

[7] Novoselov K S, Geim A K, Morozov S V, Jiang D, Katsnelson M I, Grigorieva I V, Dubonos S V and Firsov A A 2005 Nature 438197

Zhang Y, Tang Y-W, Stormer H L and Kim P 2005 Nature 438201

[8] Geim A K and Novoselov K S 2007 Nature Mater. 6183

[9] Beneventano C G, Giacconi P, Santangelo E M and Soldati R 2007 J. Phys. A: Math. Theor. 40 F435

[10] Dowker J S and Critchley R 1976 Phys. Rev. D 133224

[11] Cognola G, Elizalde E and Zerbini S 2003 Commun. Math. Phys. 237507

[12] Beneventano C G and Santangelo E M 2006 J. Phys. A: Math. Gen. 397457

Beneventano C G and Santangelo E M 2006 J. Phys. A: Math. Gen. 396137

[13] Berry M V 1984 Proc. R. Soc. A 39245

[14] Beneventano C G and Santangelo E M 2008 J. Phys. A: Math. Theor. 41164035

[15] Ziegler K 2007 Phys. Rev. B 75233407

[16] Ludwig A W W, Fisher M P A, Shankar R and Grinstein G 1994 Phys. Rev. B 507526

Ziegler K 1997 Phys. Rev. B 5510661

Ziegler K 1998 Phys. Rev. Lett. 803113

Katsnelson M I 2006 Eur. Phys. J. B 51157

Peres N M R, Guinea F and Neto A H C 2006 Phys. Rev. B 73125411

Tworzydlo J, Trauzettel B, Titov M, Rycerz A and Beenakker C 2006 Phys. Rev. Lett. 96246802

Cserti J 2007 Phys. Rev. B 75033405

Ostrovsky P M, Gornyi I V and Mirlin A D 2006 Phys. Rev. B 74235443

Ryu S, Mudry C, Furusaki A and Ludwig A W W 2006 arXiv:cond-mat/0610598

Falkovsky L A and Varlamov A A 2006 arXiv:cond-mat/0606800

Ziegler K 2006 Phys. Rev. Lett. 97266802

Gusynin V P and Sharapov S G 2002 Phys. Rev. B 66045108

Gusynin V P and Sharapov S G 2005 Phys. Rev. Lett. 95146801

Gusynin V P and Sharapov S G 2006 Phys. Rev. B 73245411

[17] Peres N M, Guinea F and Neto A H C 2006 Phys. Rev. B 73125411

[18] Flekkøy E G and Leinaas J M 1991 Int. J. Mod. Phys. A 65327

[19] Deser S, Griguolo L and Seminara D 1998 Phys. Rev. D 577444

[20] Forte S 1992 Int. J. Mod. Phys. 71025

[21] Zak J 1964 Phys. Rev. 134 A1602 Zak J 1964 Phys. Rev. 134 A1607

[22] Luk'yanchuk I A and Kopelevich Y 2006 Phys. Rev. Lett. 97256801

[23] McCann E and Fal'ko V I 2006 Phys. Rev. Lett. 96086805

[24] Novoselov K S, McCann E, Morozov S V, Falko V I, Katsnelson M I, Zeitler U, Jiang D, Schedin F and Geim A K 2006 Nature Phys. 2177

[25] Nikishov A I 1970 Soviet Phys._JETP 30660

Nikishov A I 1969 Transl. Zh. Eksp. Teor. Fiz. 571210

Nikishov A I 2003 Transl. Zh. Eksp. Teor. Fiz. 571210 (arXiv:hep-th/0207085v2 arXiv:hep-th/0211088v2)

[26] Gradshteyn I S and Ryzhik I M 1996 Table of Integrals, Series, and Products 5th edn ed A Jeffrey (San Diego: Academic)

[27] Dunne G V 2008 arXiv:0812.3163v1 [hep-th]

[28] Klimenko K G 1992 Z. Phys. C 54323

Klimenko K G 1992 Theor. Math. Phys. 891287

Dittrich W and Gies H 1997 Phys. Lett. B 392182

Gusynin V P and Shovkovy I A 1999 J. Math. Phys. 405406

Lin Q-g 1999 J. Phys. G: Nucl. Part. Phys. 2517 
[29] Moroz S 2007 arXiv:0710.4880 [hep-ph]

[30] Allor D, Cohen T D and McGady D A 2008 arXiv:0708.1471v4 [cond-mat.mes-hall]

[31] Itzykson C and Zuber J B 1980 Quantum Field Theory (New York: McGraw-Hill)

[32] Schwinger J 1951 Phys. Rev. 82664

[33] Gavrilov S P and Gitman D M 1996 Phys. Rev. D 537162

[34] Nikishov A I 1970 Nucl. Phys. B 21346

[35] Abramowitz M and Stegun I A 1978 Handbook of Mathematical Functions with Formulas, Graphs and Mathematical Tables (New York: Dover)

[36] Gamboa Saraví R E, Muschietti M A, Schaposnik F A and Solomin J E 1985 J. Math. Phys. 262045

[37] Miao F, Wijeratne S, Zhang Y, Coskun U C, Bao W and Lau C N 2007 Science 3171530 